\title{
COMPLETELY BOUNDED MULTIPLIERS OVER LOCALLY COMPACT QUANTUM GROUPS
}

\author{
ZHIGUO HU, MATTHIAS NEUFANG, AND ZHONG-JIN RUAN
}

\begin{abstract}
In this paper, we consider several interesting multiplier algebras associated with a locally compact quantum group $\mathbb{G}$. Firstly, we study the completely bounded right multiplier algebra $M_{c b}^{r}\left(L_{1}(\mathbb{G})\right.$ ). We show that $M_{c b}^{r}\left(L_{1}(\mathbb{G})\right)$ is a dual Banach algebra with a natural operator predual $Q^{r}\left(L_{1}(\mathbb{G})\right.$ ), and the completely isometric representation of $M_{c b}^{r}\left(L_{1}(\mathbb{G})\right)$ on $\mathcal{B}\left(L_{2}(\mathbb{G})\right)$, studied recently by Junge, Neufang and Ruan, is actually weak*-weak* continuous. Secondly, we study the left uniformly continuous space $L U C(\mathbb{G})$ and its Banach algebra dual $L U C(\mathbb{G})^{*}$. We prove that $L U C(\mathbb{G})$ is a unital $\mathrm{C}^{*}$-subalgebra of $L_{\infty}(\mathbb{G})$ if the quantum group $\mathbb{G}$ is semi-regular. We show the connection between $L U C(\mathbb{G})^{*}$ and the quantum measure algebra $M(\mathbb{G})$, as well as their representations on $L_{\infty}(\mathbb{G})$ and $\mathcal{B}\left(L_{2}(\mathbb{G})\right.$ ). Finally, we study the right uniformly complete qotient space $U C Q^{r}\left(L_{1}(\mathbb{G})\right)$ and its Banach algebra dual $U C Q^{r}\left(L_{1}(\mathbb{G})\right)^{*}$. For co-amenable quanum groups $\mathbb{G}$, we obtain the weak*-homeomorphic completely isometric algebra isomorphism $M_{c b}^{r}\left(L_{1}(\mathbb{G})\right) \cong M(\mathbb{G})$ and the completely isometric isomorphism $U C Q^{r}\left(L_{1}(\mathbb{G})\right) \cong L U C(\mathbb{G})$.
\end{abstract}

\section{INTRODUCTION}

Let $\mathbb{G}=\left(L_{\infty}(\mathbb{G}), \Gamma, \varphi, \psi\right)$ be a locally compact quantum group. Then the operator predual $L_{1}(\mathbb{G})$ of $L_{\infty}(\mathbb{G})$ with the multiplication $\Gamma_{*}$ is a faithful completely contractive Banach algebra. If we let $C_{0}(\mathbb{G})$ denote the associated Hopf $\mathrm{C}^{*}$-subalgebra of $L_{\infty}(\mathbb{G})$, then its operator dual $M(\mathbb{G})=C_{0}(\mathbb{G})^{*}$ is a faithful completely contractive Banach algebra, containing $L_{1}(\mathbb{G})$ as a closed two-sided ideal. Therefore, we can study the quantum group analogue of many other Banach algebras interesting in abstract harmonic analysis, and we can develop a corresponding theory for quantum harmonic analysis. The aim of this paper is to study the following spaces associated with locally compact quantum groups $\mathbb{G}$ : (1) the completely bounded right multiplier algebra $M_{c b}^{r}\left(L_{1}(\mathbb{G})\right)$ of $L_{1}(\mathbb{G})$ and its predual $Q^{r}\left(L_{1}(\mathbb{G})\right) ;(2)$ the left uniformly continuous space $L U C(\mathbb{G})$ and its operator dual $L U C(\mathbb{G})^{*} ;(3)$ the right uniformly complete quotient space $U C Q^{r}\left(L_{1}(\mathbb{G})\right)$ and its the operator dual $U C Q^{r}\left(L_{1}(\mathbb{G})\right)^{*}$.

Date: August 02, 2009.

2000 Mathematics Subject Classification. 43A10, 43A20, 43A30, 46H05 (primary).

The first and the second authors were partially supported by NSERC. The third author was partially supported by the National Science Foundation DMS-0500535. 
In Section 2, we recall notations and definitions of locally compact quantum groups introduced by Kustermann and Vaes in [20] and [21]. We also collect some module properties for the Hopf $\mathrm{C}^{*}$-algebra $C_{0}(\mathbb{G})$ and the quantum measure algebra $M(\mathbb{G})$.

Sections 3 and 4 are devoted to the study of the completely bounded right multiplier algebra $M_{c b}^{r}\left(L_{1}(\mathbb{G})\right)$. It is shown in the recent paper [17] by Junge, Neufang, and Ruan that for any locally compact quantum group $\mathbb{G}$, there exists a completely isometric algebra isomorphism

$$
\Theta^{r}: M_{c b}^{r}\left(L_{1}(\mathbb{G})\right) \cong \mathcal{C} \mathcal{B}_{L_{\infty}(\hat{\mathbb{G}})}^{\sigma, L_{\infty}(\mathbb{G})}\left(\mathcal{B}\left(L_{2}(\mathbb{G})\right)\right)
$$

where $\mathcal{C B}_{L_{\infty}(\hat{\mathbb{G}})}^{\sigma, L_{\infty}(\mathbb{G})}\left(\mathcal{B}\left(L_{2}(\mathbb{G})\right)\right)$ is the algebra of normal completely bounded $L_{\infty}(\hat{\mathbb{G}})$-bimodule homomorphisms on $\mathcal{B}\left(L_{2}(\mathbb{G})\right)$, which map $L_{\infty}(\mathbb{G})$ into $L_{\infty}(\mathbb{G})$. We show in Theorem 3.4 and Proposition 3.5 that $M_{c b}^{r}\left(L_{1}(\mathbb{G})\right)$ is a dual Banach algebra with a natural operator predual $Q^{r}\left(L_{1}(\mathbb{G})\right)$. This generalizes the results by Haagerup and Kraus [12] on completely bounded Fourier multiplier algebra $M_{c b} A(G)$ and the results by Kraus and Ruan [19] on completely bounded Kac multiplier algebra $M_{c b} A(\mathbb{K})$. We prove in Theorem 4.1 that the isomorphism $\Theta^{r}$ in (1.1) is actually a weak* homeomorphism. This generalizes the weak*-homeomorphic representation theorems for measure algebras $M(G)$ and for completely bounded Fourier multiplier algebras $M_{c b} A(G)$ proved by Neufang, Ruan, and Spronk in [30, Theorem 3.2 and Theorem 4.5]. We also study the relation between $Q^{r}\left(L_{1}(\mathbb{G})\right)$ and $C_{0}(\mathbb{G})$, and obtain the weak*-weak* continuity of the canonical embedding $M(\mathbb{G}) \rightarrow M_{c b}^{r}\left(L_{1}(\mathbb{G})\right)$. This leads to some characterizations of co-amenable quantum groups, generalizing results in $[19, \S 7]$ for Kac algebras to locally compact quantum groups. Since the argument for completely bounded left (respectively, double) multiplier algebra is quite similar, we only remark briefly the corresponding results at the end of Section 4.

Section 5 is focused on the left uniformly continuous space $L U C(\mathbb{G})$ (respectively, the right uniformly continuous space $R U C(\mathbb{G})$ ) on a locally compact quantum group $\mathbb{G}$. In the case of the commutative quantum group $\mathbb{G}_{a}=L_{\infty}(G)$, where $G$ is a locally compact group, the space $L U C\left(\mathbb{G}_{a}\right)$ is the usual space $L U C(G)$ of bounded left uniformly continuous functions on $G$. On the other hand, if we consider $\mathbb{G}_{s}=L(G)$, the dual quantum group of $\mathbb{G}_{a}$, then $L U C\left(\mathbb{G}_{s}\right)$ is the space $U C B(\hat{G})$ of uniformly continuous linear functionals on $A(G)$ introduced by Granirer [10]. It is known that both $L U C(G)$ and $U C B(\hat{G})$ are unital $\mathrm{C}^{*}$-algebras. It is also easy to see that if $\mathbb{G}$ is a discrete (respectively, compact) quantum group, then $L U C(\mathbb{G})=L_{\infty}(\mathbb{G})$ (respectively, $L U C(\mathbb{G})=C_{0}(\mathbb{G})$ ) is a unital $\mathrm{C}^{*}$-algebra. However, it is still an open question whether $\operatorname{LUC}(\mathbb{G})$ is a $\mathrm{C}^{*}$-algebra for an arbitrary locally compact quantum group $\mathbb{G}$. Runde showed in $[37]$ that $L U C(\mathbb{G})$ is an operator system in $L_{\infty}(\mathbb{G})$ such that

$$
C_{0}(\mathbb{G}) \subseteq L U C(\mathbb{G}) \subseteq M\left(C_{0}(\mathbb{G})\right)
$$

where $M\left(C_{0}(\mathbb{G})\right) \subseteq L_{\infty}(\mathbb{G})$ denotes the multiplier algebra of $C_{0}(\mathbb{G})$. He further showed that $L U C(\mathbb{G})$ is a unital $\mathrm{C}^{*}$-subalgebra of $M\left(C_{0}(\mathbb{G})\right)$ if $\mathbb{G}$ is co-amenable and $C_{0}(\mathbb{G})$ has a bounded approximate identity 
contained in the centre of $C_{0}(\mathbb{G})$. Moreover, Salmi [38] introduced a left uniformly continuous $\mathrm{C}^{*}$-algebra $\mathcal{L U C}(\mathbb{G})$ by a different $\mathrm{C}^{*}$-algebra approach. Results in [38] indicate that if $\mathbb{G}$ is co-amenable and the associated right fundamental unitary operator $V$ is regular, then our $L U C(\mathbb{G})$ is equal to $\mathcal{L U C}(\mathbb{G})$ and thus is a unital $\mathrm{C}^{*}$-algebra. Our main result Theorem 5.6 in Section 5 shows that for the large class of semi-regular locally compact quantum groups $\mathbb{G}$, which includes Kac algebras, $L U C(\mathbb{G})$ and $R U C(\mathbb{G})$ are indeed unital $\mathrm{C}^{*}$-subalgebras of $M\left(C_{0}(\mathbb{G})\right)$.

Given a locally compact quantum group $\mathbb{G}$, the Arens products on $L_{\infty}(\mathbb{G})^{*}$ induce a natural completely contractive Banach algebra structure on $\operatorname{LUC}(\mathbb{G})^{*}$ and $R U C(\mathbb{G})^{*}$, respectively. We study in Section 6 the Banach algebras $L U C(\mathbb{G})^{*}$ and $R U C(\mathbb{G})^{*}$, their relations with the quantum measure algebra $M(\mathbb{G})$, and characterizations of co-amenable quantum groups in terms of representations of these algebras. We shall focus our study on $\operatorname{LUC}(\mathbb{G})^{*}$. The corresponding results for $R U C(\mathbb{G})^{*}$ can be obtained analogously.

In Section 7 , we introduce the right uniformly quotient space $U C Q^{r}\left(L_{1}(\mathbb{G})\right)$ and its operator dual $U C Q^{r}\left(L_{1}(\mathbb{G})\right)^{*}$. We show that there is a natural completely contractive Banach algebra structure on $U C Q^{r}\left(L_{1}(\mathbb{G})\right)^{*}$, and we study the completely isometric representation of $U C Q^{r}\left(L_{1}(\mathbb{G})\right)^{*}$ on $\mathcal{B}\left(L_{2}(\mathbb{G})\right)$. We show in Theorem 7.3 that if $\mathbb{G}$ is co-amenable, then $U C Q^{r}\left(L_{1}(\mathbb{G})\right)=L U C(\mathbb{G})$ completely isometrically. In particular, if $\mathbb{G}_{a}$ and $\mathbb{G}_{s}$ are the commutative and the co-commutative quantum groups associated with a locally compact group $G$, respectively, then we have $U C Q^{r}\left(L_{1}\left(\mathbb{G}_{a}\right)\right)=L U C(G)$, and $U C Q^{r}\left(L_{1}\left(\mathbb{G}_{s}\right)\right)$ is the right-hand side version of the uniformly complete quotient space $U C Q(G)$ introduced (via the left fundamental unitary operator $W$ ) by Popa and Ruan in [33]. Therefore, results obtained in this section unify and generalize some results on $\operatorname{LUC}(G)^{*}$ and $U C Q(G)^{*}$ in [29], [30], and [33].

\section{Definitions AND PRELIMINARY RESUlts}

Let us first recall from [21] and [39] that a (von Neumann algebraic) locally compact quantum group is a quatriple $\mathbb{G}=\left(L_{\infty}(\mathbb{G}), \Gamma, \varphi, \psi\right)$, where $L_{\infty}(\mathbb{G})$ is a Hopf von Neumann algebra with a co-associative comultiplication $\Gamma: L_{\infty}(\mathbb{G}) \rightarrow L_{\infty}(\mathbb{G}) \bar{\otimes} L_{\infty}(\mathbb{G})$, and $\varphi$ and $\psi$ are normal faithful left and right Haar weights on $L_{\infty}(\mathbb{G})$, respectively. For each locally compact quantum group $\mathbb{G}$, there exist a left fundamental unitary operator $W$ on $L_{2}(\mathbb{G}, \varphi) \otimes L_{2}(\mathbb{G}, \varphi)$ and a right fundamental unitary operator $V$ on $L_{2}(\mathbb{G}, \psi) \otimes L_{2}(\mathbb{G}, \psi)$ which satisfy the pentagonal relation; that is,

$$
W_{12} W_{13} W_{23}=W_{23} W_{12} \text { and } V_{12} V_{13} V_{23}=V_{23} V_{12} \text {. }
$$

The co-multiplication $\Gamma$ on $L_{\infty}(\mathbb{G})$ can be expressed as

$$
\Gamma(x)=W^{*}(1 \otimes x) W=V(x \otimes 1) V^{*}
$$


for all $x \in L_{\infty}(\mathbb{G})$. We identify $L_{2}(\mathbb{G}, \varphi)$ and $L_{2}(\mathbb{G}, \psi)$, and simply use $L_{2}(\mathbb{G})$ for this Hilbert space in the rest of this paper. In this case, there exists a unitary operator $U$ on $L_{2}(\mathbb{G})$ such that

$$
V=\Sigma(1 \otimes U) W\left(1 \otimes U^{*}\right) \Sigma,
$$

where $\Sigma$ is the flip operator on $L_{2}(\mathbb{G}) \otimes L_{2}(\mathbb{G})$ given by $\Sigma(\xi \otimes \eta)=\eta \otimes \xi\left(\xi, \eta \in L_{2}(\mathbb{G})\right)$.

The pre-adjoint of $\Gamma$ induces an associative completely contractive multiplication

$$
\star: f_{1} \otimes f_{2} \in L_{1}(\mathbb{G}) \hat{\otimes} L_{1}(\mathbb{G}) \rightarrow f_{1} \star f_{2}=\left(f_{1} \otimes f_{2}\right) \circ \Gamma \in L_{1}(\mathbb{G})
$$

on $L_{1}(\mathbb{G})=L_{\infty}(\mathbb{G})_{*}$. It is known from [13, Proposition 1] that the multiplication $\star$ is faithful in the sense that for any $f \in L_{1}(\mathbb{G})$, we have $f=0$ if $f \star g=0$ (respectively, $g \star f=0$ ) for all $g \in L_{1}(\mathbb{G})$. Let $\left\langle L_{1}(\mathbb{G}) \star L_{1}(\mathbb{G})\right\rangle$ denote the closed linear span of $f \star g$ with $f, g \in L_{1}(\mathbb{G})$. Then we have

$$
\left\langle L_{1}(\mathbb{G}) \star L_{1}(\mathbb{G})\right\rangle=L_{1}(\mathbb{G}),
$$

since the multiplication $\star$ is a complete quotient from $L_{1}(\mathbb{G}) \hat{\otimes} L_{1}(\mathbb{G})$ onto $L_{1}(\mathbb{G})$. A locally compact quantum group $\mathbb{G}$ is called co-amenable if $L_{1}(\mathbb{G})$ has a bounded left (respectively, right, or two-sided) approximate identity. It is shown in [13] that $\mathbb{G}$ is co-amenable if and only if $L_{1}(\mathbb{G})$ has an approximate identity, which consists of normal states on $L_{\infty}(\mathbb{G})$.

If $G$ is a locally compact group, we let $\mathbb{G}_{a}=\left(L_{\infty}(G), \Gamma_{a}, \varphi_{a}, \psi_{a}\right)$ denote the commutative quantum group associated with the commutative Hopf von Neumann algebra $L_{\infty}(G)$ with the co-multiplication $\Gamma_{a}(f)(s, t)=f(s t)$. The dual quantum group $\hat{\mathbb{G}}_{a}$ of $\mathbb{G}_{a}$ is the co-commutative quantum group $\mathbb{G}_{s}=$ $\left(L(G), \Gamma_{s}, \varphi_{s}, \psi_{s}\right)$, where $L(G)$ is the left group von Neumann algebra with the co-multiplication $\Gamma_{s}(\lambda(t))=$ $\lambda(t) \otimes \lambda(t)$. We can also consider the dual quantum group $\hat{\mathbb{G}}_{a}^{\prime}=\mathbb{G}_{s}^{\prime}$ associated with the right group von Neumann algebra $R(G)$ and the co-multiplication $\Gamma_{s}^{\prime}(\rho(t))=\rho(t) \otimes \rho(t)$. In this case, $L_{1}\left(\mathbb{G}_{a}\right)$ is the usual convolution algebra $L_{1}(G)$, and $L_{1}\left(\mathbb{G}_{s}\right)=L_{1}\left(\mathbb{G}_{s}^{\prime}\right)$ is the Fourier algebra $A(G)$. Therefore, the commutative quantum group $\mathbb{G}_{a}$ is always co-amenable, and the co-commutative quantum group $\mathbb{G}_{s}$ (respectively, $\mathbb{G}_{s}^{\prime}$ ) is co-amenable if and only if the associated group $G$ is amenable.

Given a locally compact quantum group $\mathbb{G}$, we can obtain the left regular representation $\lambda: L_{1}(\mathbb{G}) \rightarrow$ $\mathcal{B}\left(L_{2}(\mathbb{G})\right)$ defined by

$$
\lambda: f \in L_{1}(\mathbb{G}) \rightarrow \lambda(f)=(f \otimes \iota)(W) \in \mathcal{B}\left(L_{2}(\mathbb{G})\right),
$$

which is an injective completely contractive algebra homomorphism from $L_{1}(\mathbb{G})$ into $\mathcal{B}\left(L_{2}(\mathbb{G})\right)$. Then $L_{\infty}(\hat{\mathbb{G}})=\left\{\lambda(f): f \in L_{1}(\mathbb{G})\right\}^{\prime \prime}$ is the Hopf von Neumann algebra in the dual quantum group $\hat{\mathbb{G}}$. Correspondingly, we can obtain the right regular representation $\rho: L_{1}(\mathbb{G}) \rightarrow \mathcal{B}\left(L_{2}(\mathbb{G})\right)$ defined by

$$
\rho: f \in L_{1}(\mathbb{G}) \rightarrow \rho(f)=(\iota \otimes f)(V) \in \mathcal{B}\left(L_{2}(\mathbb{G})\right),
$$

which is also an injective completely contractive algebra homomorphism from $L_{1}(\mathbb{G})$ into $\mathcal{B}\left(L_{2}(\mathbb{G})\right)$. Then $L_{\infty}\left(\hat{\mathbb{G}}^{\prime}\right)=\left\{\rho(f): f \in L_{1}(\mathbb{G})\right\}^{\prime \prime}$ is the Hopf von Neumann algebra in the dual quantum group $\hat{\mathbb{G}}^{\prime}$. It follows 
that $W \in L_{\infty}(\mathbb{G}) \bar{\otimes} L_{\infty}(\hat{\mathbb{G}})$ and $V \in L_{\infty}\left(\hat{\mathbb{G}}^{\prime}\right) \bar{\otimes} L_{\infty}(\mathbb{G})$. We can conclude from $(2.3)$ that

$$
\rho(f)=U \lambda(f) U^{*}
$$

for all $f \in L_{1}(\mathbb{G})$.

We can also define the completely contractive injections

$$
\lambda_{*}: \hat{f} \in L_{1}(\hat{\mathbb{G}}) \rightarrow \lambda_{*}(\hat{f})=(\iota \otimes \hat{f})(W) \in L_{\infty}(\mathbb{G})
$$

and

$$
\rho_{*}: \hat{f}^{\prime} \in L_{1}\left(\hat{\mathbb{G}}^{\prime}\right) \rightarrow \rho_{*}\left(\hat{f}^{\prime}\right)=\left(\hat{f}^{\prime} \otimes \iota\right)(V) \in L_{\infty}(\mathbb{G}) .
$$

In fact, we have

$$
\left\langle\lambda_{*}(\hat{f}), f\right\rangle=\langle\hat{f}, \lambda(f)\rangle \text { and }\left\langle\rho_{*}\left(\hat{f}^{\prime}\right), f\right\rangle=\left\langle\hat{f}^{\prime}, \rho(f)\right\rangle
$$

for all $f \in L_{1}(\mathbb{G}), \hat{f} \in L_{1}(\hat{\mathbb{G}})$, and $\hat{f}^{\prime} \in L_{1}\left(\hat{\mathbb{G}}^{\prime}\right)$. We then obtain the corresponding Hopf $\mathrm{C}^{*}$-subalgebra

$$
C_{0}(\mathbb{G})=\overline{\lambda_{*}\left(L_{1}(\hat{\mathbb{G}})\right)}\|\cdot\| \overline{\rho_{*}\left(L_{1}\left(\hat{\mathbb{G}}^{\prime}\right)\right)}\|\cdot\|
$$

of $L_{\infty}(\mathbb{G})$ with the co-multiplication

$$
\Gamma: x \in C_{0}(\mathbb{G}) \rightarrow \Gamma(x)=V(x \otimes 1) V^{*} \in M\left(C_{0}(\mathbb{G}) \otimes C_{0}(\mathbb{G})\right),
$$

where $M\left(C_{0}(\mathbb{G}) \otimes C_{0}(\mathbb{G})\right)$ is the multiplier algebra of the $\mathrm{C}^{*}$-algebra $C_{0}(\mathbb{G}) \otimes C_{0}(\mathbb{G})$. It is known that for any locally compact quantum group $\mathbb{G}$,

$$
\Gamma\left(C_{0}(\mathbb{G})\right)\left(C_{0}(\mathbb{G}) \otimes 1\right) \text { and } \Gamma\left(C_{0}(\mathbb{G})\right)\left(1 \otimes C_{0}(\mathbb{G})\right)
$$

are norm dense in $C_{0}(\mathbb{G}) \otimes C_{0}(\mathbb{G})$ (cf. [21, Corollary 6.11]).

Let $M(\mathbb{G})=C_{0}(\mathbb{G})^{*}$ denote the operator dual of $C_{0}(\mathbb{G})$. Then $M(\mathbb{G})$ is a completely contractive $C_{0}(\mathbb{G})$-bimodule with the module operation given by

$$
\langle\mu \bullet x, y\rangle=\langle\mu, x y\rangle \text { and }\langle x \bullet \mu, y\rangle=\langle\mu, y x\rangle
$$

for $\mu \in M(\mathbb{G})$ and $x, y \in C_{0}(\mathbb{G})$. Since the linear span of $\mu \bullet x$ (respectively, $x \bullet \mu$ ) with $\mu \in M(\mathbb{G})$ and $x \in C_{0}(\mathbb{G})$ is norm dense in $M(\mathbb{G})$, we can obtain the following proposition by Cohen's factorization theorem and the fact that $C_{0}(\mathbb{G})$ is weak* dense in $L_{\infty}(\mathbb{G})$.

Proposition 2.1. Let $\mathbb{G}$ be a locally compact quantum group. Then the following assertions holds.

(1) $M(\mathbb{G})$ is a $C_{0}(\mathbb{G})$-bimodule with $M(\mathbb{G})=M(\mathbb{G}) \bullet C_{0}(\mathbb{G})=C_{0}(\mathbb{G}) \bullet M(\mathbb{G})$.

(2) $L_{1}(\mathbb{G})$ is a $C_{0}(\mathbb{G})$-bimodule with $L_{1}(\mathbb{G})=L_{1}(\mathbb{G}) \bullet C_{0}(\mathbb{G})=C_{0}(\mathbb{G}) \bullet L_{1}(\mathbb{G})$. 
Suppose that $\mu \in M(\mathbb{G})=C_{0}(\mathbb{G})^{*}$. It is known that $\mu$ has a unique strictly continuous extension $\tilde{\mu} \in M\left(C_{0}(\mathbb{G})\right)^{*}$. It is also known that for any $\mathrm{C}^{*}$-algebra $B$, the right slice map $\mu \otimes \iota: C_{0}(\mathbb{G}) \otimes B \rightarrow B$ is completely bounded and has a unique extension

$$
\widetilde{\mu \otimes \iota}: M\left(C_{0}(\mathbb{G}) \otimes B\right) \rightarrow M(B)
$$

that is strictly continuous on the unit ball of $M\left(C_{0}(\mathbb{G}) \otimes B\right.$ ) (cf. [2, Theorem 2.1]). This is also true for the left slice map $\iota \otimes \mu: B \otimes C_{0}(\mathbb{G}) \rightarrow B$. Therefore, for all $\mu, \nu \in M(\mathbb{G})$, we have

$$
(\widetilde{\mu \otimes \nu}) \circ \Gamma=\tilde{\mu}(\widetilde{\iota \otimes \nu}) \circ \Gamma=\tilde{\nu}(\widetilde{\mu \otimes \iota}) \circ \Gamma
$$

(cf. [2, Proposition 2.2]). If there is no confusion, we will omit the symbol " " for these extensions. Clearly, these extensions are norm preserving. Note that $(\mu \otimes \iota) \Gamma(x) \in C_{0}(\mathbb{G})$ and $(\iota \otimes \mu) \Gamma(x) \in C_{0}(\mathbb{G})$ for $\mu \in M(\mathbb{G})$ and $x \in C_{0}(\mathbb{G})$ (cf. [20, Definition 4.1]). Then we can express (2.7) as

$$
\mu \star \nu=\mu \square \nu=\mu \diamond \nu .
$$

See Section 6 for the precise meaning of the products $\square$ and $\diamond$.

Thus $(\mu, \nu) \mapsto(\mu \otimes \nu) \circ \Gamma$ defines a completely contractive multiplication $\star$ on $M(\mathbb{G})$. With this multiplication, $M(\mathbb{G})$ is a completely contractive dual Banach algebra (i.e., the multiplication on $M(\mathbb{G})$ is separately weak*-weak* continuous), and $M(\mathbb{G})$ contains $L_{1}(\mathbb{G})$ as a norm closed two-sided ideal.

For $x \in C_{0}(\mathbb{G})$ and $\mu \in M(\mathbb{G})$, by $(2.7)$, we get

$$
\mu \star x=(\iota \otimes \mu) \Gamma(x) \in C_{0}(\mathbb{G}) \text { and } x \star \mu=(\mu \otimes \iota) \Gamma(x) \in C_{0}(\mathbb{G}) .
$$

We have the following proposition on the canonical $M(\mathbb{G})$-bimodule action on $C_{0}(\mathbb{G})$.

Proposition 2.2. Let $\mathbb{G}$ be a locally compact quantum group. Then $C_{0}(\mathbb{G})$ is an operator $M(\mathbb{G})$-bimodule such that

$$
C_{0}(\mathbb{G})=\left\langle L_{1}(\mathbb{G}) \star C_{0}(\mathbb{G})\right\rangle=\left\langle M(\mathbb{G}) \star C_{0}(\mathbb{G})\right\rangle=\left\langle C_{0}(\mathbb{G}) \star L_{1}(\mathbb{G})\right\rangle=\left\langle C_{0}(\mathbb{G}) \star M(\mathbb{G})\right\rangle .
$$

Therefore, the multiplication $\star$ on $M(\mathbb{G})$ is faithful, and $C_{0}(\mathbb{G})$ can be expressed as

$$
C_{0}(\mathbb{G})=\left\langle L_{1}(\mathbb{G}) \star L_{1}\left(\hat{G}^{\prime}\right)\right\rangle=\left\langle L_{1}\left(\hat{G}^{\prime}\right) \star L_{1}(\mathbb{G})\right\rangle
$$

via the completely contractive injection $\rho_{*}: L_{1}\left(\hat{G}^{\prime}\right) \rightarrow C_{0}(\mathbb{G})$.

Proof. It suffices to show that the linear span of $f \star x$ (respectively, $x \star f$ ) with $f \in L_{1}(\mathbb{G})$ and $x \in C_{0}(\mathbb{G})$ is norm dense in $C_{0}(\mathbb{G})$. This follows from the Hahn-Banach theorem and the fact that the set $\{\Gamma(x)(1 \otimes y)$ : $\left.x, y \in C_{0}(\mathbb{G})\right\}$ (respectively, $\left\{\Gamma(x)(y \otimes 1): x, y \in C_{0}(\mathbb{G})\right\}$ ) is norm dense in $C_{0}(\mathbb{G}) \otimes C_{0}(\mathbb{G})$. Indeed, if $\mu \in M(\mathbb{G})$ and $\mu(f \star x)=0$ for all $f \in L_{1}(\mathbb{G})$ and $x \in C_{0}(\mathbb{G})$, then for any $y \in C_{0}(\mathbb{G})$, we have

$$
\langle\mu \otimes f, \Gamma(x)(1 \otimes y)\rangle=\mu((y \bullet f) \star x)=0 .
$$


This shows that $C_{0}(\mathbb{G})=\left\langle L_{1}(\mathbb{G}) \star C_{0}(\mathbb{G})\right\rangle$. We can similarly prove that $C_{0}(\mathbb{G})=\left\langle C_{0}(\mathbb{G}) \star L_{1}(\mathbb{G})\right\rangle$.

Remark 2.3. It was shown by Bédos and Tuset [3] that a locally compact quantum group $\mathbb{G}$ is coamenable if and only if $M(\mathbb{G})$ is unital. Therefore, when $\mathbb{G}$ is co-amenable, we have $M(\mathbb{G}) \star M(\mathbb{G})=M(\mathbb{G})$. However, it seems open whether we have $\langle M(\mathbb{G}) \star M(\mathbb{G})\rangle=M(\mathbb{G})$ for non-co-amenable quantum groups $\mathbb{G}$ with $M(\mathbb{G}) \neq L_{1}(\mathbb{G})($ cf. $(2.5))$.

If $G$ is a locally compact group, then $C_{0}\left(\mathbb{G}_{a}\right)=C_{0}(G)$ is the space of continuous functions on $G$ vanishing at infinity, and $M\left(\mathbb{G}_{a}\right)=M(G)$ is the measure algebra of $G$. Correspondingly, $C_{0}\left(\hat{\mathbb{G}}_{a}\right)=C_{\lambda}^{*}(G)$ is the left group $\mathrm{C}^{*}$-algebra (respectively, $C_{0}\left(\hat{\mathbb{G}}_{a}^{\prime}\right)=C_{\rho}^{*}(G)$ is the right group $\mathrm{C}^{*}$-algebra) of $G$, and we have $M\left(\hat{\mathbb{G}}_{a}\right)=B_{\lambda}(G)$ (respectively, $M\left(\hat{\mathbb{G}}_{a}^{\prime}\right)=B_{\rho}(G)$ ).

To end this section, we note that there is a universal quantum group $\mathrm{C}^{*}$-algebra $C_{u}(\mathbb{G})$ such that its dual space $C_{u}(G)^{*}$ is a completely contractive unital Banach algebra, containing $M(\mathbb{G})$ as a norm closed two-sided ideal. For the commutative quantum group $\mathbb{G}_{a}$, we have $C_{u}\left(\mathbb{G}_{a}\right)=C_{0}(G)$; for the cocommutative quantum group $\mathbb{G}_{s}$, we have $C_{u}\left(\mathbb{G}_{s}\right)=C^{*}(G)$, the full group $\mathrm{C}^{*}$-algebra of $G$. It is known that $\mathbb{G}$ is co-amenable if and only if $M(\mathbb{G})=C_{u}(\mathbb{G})^{*}$ (respectively, $C_{0}(\mathbb{G})=C_{u}(\mathbb{G})$ ). The reader is referred to [1] and [3] for details.

\section{The Dual Banach Algebra structure on $M_{c b}^{r}\left(L_{1}(\mathbb{G})\right)$}

Let $\mathbb{G}$ be a locally compact quantum group. Let us first recall from [16] that a linear map $T$ on $L_{1}(\mathbb{G})$ is called a right centralizer (respectively, a left centralizer) if

$$
T(f \star g)=f \star T(g) \quad \text { (respectively, } T(f \star g)=T(f) \star g)
$$

for all $f, g \in L_{1}(\mathbb{G})$. A double centralizer is a pair $(S, T)$ of maps on $L_{1}(\mathbb{G})$ such that

$$
f \star S(g)=T(f) \star g
$$

for $f, g \in L_{1}(\mathbb{G})$. We let $C_{c b}^{r}\left(L_{1}(\mathbb{G})\right), C_{c b}^{l}\left(L_{1}(\mathbb{G})\right)$, and $C_{c b}\left(L_{1}(\mathbb{G})\right)$ denote the spaces of completely bounded right, left, and double centralizers on $L_{1}(\mathbb{G})$, respectively. We regard $C_{c b}^{r}\left(L_{1}(\mathbb{G})\right)$ and $C_{c b}^{l}\left(L_{1}(\mathbb{G})\right)$ as operator subspaces of $\mathcal{C B}\left(L_{1}(\mathbb{G})\right)$. Then $C_{c b}^{r}\left(L_{1}(\mathbb{G})\right)$ and $C_{c b}^{l}\left(L_{1}(\mathbb{G})\right)$ are unital (and thus faithful) completely contractive Banach algebras with multiplication given by the opposite composition and the composition, respectively. The operator space matrix norm on $C_{c b}\left(L_{1}(\mathbb{G})\right)$ is given by

$$
\left\|\left[\left(S_{i j}, T_{i j}\right)\right]\right\|_{c b}=\max \left\{\left\|\left[S_{i j}\right]\right\|_{c b},\left\|\left[T_{i j}\right]\right\|_{c b}\right\}
$$

and the multiplication is given by

$$
\left(S_{1}, T_{1}\right) \circ\left(S_{2}, T_{2}\right)=\left(S_{1} \circ S_{2}, T_{2}, \circ T_{1}\right) .
$$


For each $\mu \in M(\mathbb{G})$, we can obtain the left and right multiplication maps

$$
m_{\mu}^{l}(f)=\mu \star f \quad \text { and } \quad m_{\mu}^{r}(f)=f \star \mu
$$

on $L_{1}(\mathbb{G})$. It is easy to see that $\left(m_{\mu}^{l}, m_{\mu}^{r}\right)$ is a double centralizer on $L_{1}(\mathbb{G})$ such that

$$
\left\|\left(m_{\mu}^{l}, m_{\mu}^{r}\right)\right\|_{c b}=\max \left\{\left\|m_{\mu}^{l}\right\|_{c b},\left\|m_{\mu}^{r}\right\|_{c b}\right\} \leq\|\mu\| .
$$

This defines an injective completely contractive algebra homomorphism from $M(\mathbb{G})$ into $C_{c b}^{r}\left(L_{1}(\mathbb{G})\right)$, $C_{c b}^{l}\left(L_{1}(\mathbb{G})\right)$, and $\left.C_{c b}\left(L_{1}(\mathbb{G})\right)\right)$, respectively.

Proposition 3.1. Let $\mathbb{G}$ be a locally compact quantum group. Then $\mathbb{G}$ is co-amenable if and only if we have the completely isometric algebra isomorphisms

$$
C_{c b}^{l}\left(L_{1}(\mathbb{G})\right)=M(\mathbb{G})=C_{c b}^{r}\left(L_{1}(\mathbb{G})\right)
$$

via the maps $m^{l}$ and $m^{r}$, respectively. When $\mathbb{G}$ is co-amenable, we also have

$$
C_{c b}^{l}\left(L_{1}(\mathbb{G})\right)=C_{c b}\left(L_{1}(\mathbb{G})\right)=C_{c b}^{r}\left(L_{1}(\mathbb{G})\right) .
$$

Proof. If (3.1) holds, then $M(\mathbb{G})$ is unital and thus $\mathbb{G}$ is co-amenable (cf. [3, Theorem 3.1]).

Conversely, suppose that $\mathbb{G}$ is co-amenable. Then $L_{1}(\mathbb{G})$ has a contractive approximate identity $\left\{f_{\alpha}\right\}$. Let

$$
m^{r}: \mu \in M(\mathbb{G}) \rightarrow m_{\mu}^{r} \in C_{c b}^{r}\left(L_{1}(\mathbb{G})\right)
$$

be the completely contractive algebra homomorphism, and $T \in C_{c b}^{r}\left(L_{1}(\mathbb{G})\right)$. We may assume that the net $\left\{T\left(f_{\alpha}\right)\right\}$ has a weak*-limit $\mu \in M(\mathbb{G})$ with $\|\mu\| \leq\|T\| \leq\|T\|_{c b}$. Then we have $T=m_{\mu}^{r}$, since

$$
\begin{aligned}
\left\langle m_{\mu}^{r}(f), x\right\rangle & =\langle f \star \mu, x\rangle=\langle\mu, x \star f\rangle=\lim _{\alpha}\left\langle T\left(f_{\alpha}\right), x \star f\right\rangle \\
& =\lim _{\alpha}\left\langle f \star T\left(f_{\alpha}\right), x\right\rangle=\lim _{\alpha}\left\langle T\left(f \star f_{\alpha}\right), x\right\rangle=\langle T(f), x\rangle
\end{aligned}
$$

for all $f \in L_{1}(\mathbb{G})$ and $x \in C_{0}(\mathbb{G})$. This shows that $m^{r}$ is an isometric algebra isomorphism from $M(\mathbb{G})$ onto $C_{c b}^{r}\left(L_{1}(\mathbb{G})\right)$. By a standard matricial argument, it is easy to see that $m^{r}$ is completely isometric. Similarly, we have the completely isometric algebra isomorphism $M(\mathbb{G})=C_{c b}^{l}\left(L_{1}(\mathbb{G})\right)$ via $m^{l}$.

Now if $(S, T)$ is a double centralizer in $C_{c b}\left(L_{1}(\mathbb{G})\right)$, then $T \in C_{c b}^{r}\left(L_{1}(\mathbb{G})\right)$ and thus $T=m_{\mu}^{r}$ for some $\mu \in M(\mathbb{G})$. In this case, we must have $S=m_{\mu}^{l}$, since

$$
f \star S(g)=T(f) \star g=m_{\mu}^{r}(f) \star g=(f \star \mu) \star g=f \star(\mu \star g)=f \star m_{\mu}^{l}(g)
$$

for all $f, g \in L_{1}(\mathbb{G})$ and the multiplication $\star$ on $L_{1}(\mathbb{G})$ is faithful. This shows that the map

$$
m: \mu \in M(\mathbb{G}) \rightarrow\left(m_{\mu}^{l}, m_{\mu}^{r}\right) \in C_{c b}\left(L_{1}(\mathbb{G})\right)
$$

is a completely isometric algebra isomorphism from $M(\mathbb{G})$ onto $C_{c b}\left(L_{1}(\mathbb{G})\right)$, and thus (3.2) holds. 
In the rest of this section and in Section 4, we will mainly study results related to completely bounded right centralizers. The corresponding results can be obtained analogously for completely bounded left or double centralizers, which will be mentioned briefly at the end of Section 4.

Let us recall from [17] that the adjoint mapping

$$
T \in C_{c b}^{r}\left(L_{1}(\mathbb{G})\right) \rightarrow T^{*} \in \mathcal{C B}_{L_{1}(\mathbb{G})}^{\sigma}\left(L_{\infty}(\mathbb{G})\right)
$$

defines a completely isometric algebra isomorphism from $C_{c b}^{r}\left(L_{1}(\mathbb{G})\right)$ onto the algebra $\mathcal{C B}_{L_{1}(\mathbb{G})}^{\sigma}\left(L_{\infty}(\mathbb{G})\right)$ of normal completely bounded right $L_{1}(\mathbb{G})$-module homomorphisms on $L_{\infty}(\mathbb{G})$. Moreover, it was shown in [17] that each $T \in C_{c b}^{r}\left(L_{1}(\mathbb{G})\right)$ is uniquely associated with a completely bounded right multiplier $\hat{b}^{\prime}$ in $L_{\infty}\left(\hat{\mathbb{G}}^{\prime}\right)$, for which we have $\rho(f) \hat{b}^{\prime} \in \rho\left(L_{1}(\mathbb{G})\right)$ for all $f \in L_{1}(\mathbb{G})$, the map

$$
m_{\hat{b}^{\prime}}^{r}: f \in L_{1}(\mathbb{G}) \rightarrow \rho^{-1}\left(\rho(f) \hat{b}^{\prime}\right) \in L_{1}(\mathbb{G})
$$

is completely bounded, and $T=m_{\hat{b}^{\prime}}^{r}$. Therefore, we may regard $\hat{b}^{\prime}$ as a concrete realization of the right centralizer $T$. This can be regarded as the quantum group analogue of Wendel's theorem [40] for centralizers on convolution algebras $L_{1}(G)$ and Losert's theorem [27] for centralizers on Fourier algebras $A(G)$, where $G$ is a locally compact group.

We let $M_{c b}^{r}\left(L_{1}(\mathbb{G})\right)$ denote the space of completely bounded right multipliers on $L_{1}(\mathbb{G})$. Then $M_{c b}^{r}\left(L_{1}(\mathbb{G})\right)$ is a unital subalgebra of $L_{\infty}\left(\hat{\mathbb{G}}^{\prime}\right)$. Clearly, $\rho\left(L_{1}(\mathbb{G})\right) \subseteq M_{c b}^{r}\left(L_{1}(\mathbb{G})\right)$, and $m_{\rho(f)}^{r}=m_{f}^{r}$ for $f \in L_{1}(\mathbb{G})$. We can completely isometrically and algebraically identify $C_{c b}^{r}\left(L_{1}(\mathbb{G})\right)$ with $M_{c b}^{r}\left(L_{1}(\mathbb{G})\right)$ if $M_{c b}^{r}\left(L_{1}(\mathbb{G})\right)$ is equipped with the operator space matrix norm from $C_{c b}^{r}\left(L_{1}(\mathbb{G})\right)$, i.e., we let

$$
\left\|\left[\hat{b}_{i j}^{\prime}\right]\right\|_{M_{n}\left(M_{c b}^{r}\left(L_{1}(\mathbb{G})\right)\right)}=\left\|\left[m_{\hat{b}_{i j}^{\prime}}^{r}\right]\right\|_{c b}
$$

Remark 3.2. We note that double (respectively, right and left) multipliers for Kac algebras $\mathbb{K}$ have been studied by Kraus and Ruan in [19], where they obtained a representation of bounded double centralizers (respectively, completely bounded double centralizers) as closed and densely defined operators (respectively, bounded operators) on a dense subspace of $L_{2}(\mathbb{K})$. The existence of a nice antipode $\kappa$ on $\mathbb{K}$ plays an important role in their proof. Using the representation theorem in [17], we are able to prove the above much stronger result on one-sided (i.e., right or left) completely bounded centralizers over general locally compact quantum groups.

The following proposition is the quantum group analogue of [19, Proposition 5.8]. We include the proof here for the convenience of the reader.

Proposition 3.3. Let $\mathbb{G}$ be a locally compact quantum group. Then, for each $n \in \mathbb{N}$, the closed unit ball of $M_{n}\left(M_{c b}^{r}\left(L_{1}(\mathbb{G})\right)\right)$ is weak $k^{*}$ closed in $M_{n}\left(L_{\infty}\left(\hat{\mathbb{G}}^{\prime}\right)\right)$. 
Proof. We first consider the case when $n=1$. Let $\left\{\hat{b}_{i}^{\prime}\right\}$ be a net in the closed unit ball $M_{c b}^{r}\left(L_{1}(\mathbb{G})\right)_{1}$ of $M_{c b}^{r}\left(L_{1}(\mathbb{G})\right)$ such that $\hat{b}_{i}^{\prime} \rightarrow \hat{b}^{\prime} \in L_{\infty}\left(\hat{\mathbb{G}}^{\prime}\right)$ in the weak* topology. Then $\rho(f) \hat{b}_{i}^{\prime} \rightarrow \rho(f) \hat{b}^{\prime}$ in the weak* topology for all $f \in L_{1}(\mathbb{G})$, and we have

$$
\left\langle\rho_{*}\left(\hat{b}_{i}^{\prime} \cdot \hat{f}^{\prime}\right), f\right\rangle=\left\langle\hat{b}_{i}^{\prime} \cdot \hat{f}^{\prime}, \rho(f)\right\rangle=\left\langle\rho(f) \hat{b}_{i}^{\prime}, \hat{f}^{\prime}\right\rangle \rightarrow\left\langle\rho(f) \hat{b}^{\prime}, \hat{f}^{\prime}\right\rangle=\left\langle\hat{b}^{\prime} \cdot \hat{f}^{\prime}, \rho(f)\right\rangle=\left\langle\rho_{*}\left(\hat{b}^{\prime} \cdot \hat{f}^{\prime}\right), f\right\rangle
$$

for all $\hat{f}^{\prime} \in L_{1}\left(\hat{\mathbb{G}}^{\prime}\right)$ and $f \in L_{1}(\mathbb{G})$. By [17, Proposition 4.1], $\left\|\hat{b}_{i}^{\prime}\right\|_{M_{c b}^{r}\left(L_{1}(\mathbb{G})\right)} \leq 1$ is equivalent to that

$$
\left\|\left[\rho_{*}\left(\hat{b}_{i}^{\prime} \cdot \hat{f}_{k l}^{\prime}\right)\right]\right\| \leq\left\|\left[\rho_{*}\left(\hat{f}_{k l}^{\prime}\right)\right]\right\|
$$

for all $\left[\hat{f}_{k l}^{\prime}\right] \in M_{m}\left(L_{1}\left(\hat{\mathbb{G}}^{\prime}\right)\right)$ and $m \in \mathbb{N}$. Then we can conclude from (3.4) that for any $\left[f_{s t}\right] \in M_{p}\left(L_{1}(\mathbb{G})\right)_{1}$, we have

$$
\left\|\left[\left\langle\rho_{*}\left(\hat{b} \cdot \hat{f}_{k l}^{\prime}\right), f_{s t}\right\rangle\right]\right\|=\lim _{i}\left\|\left[\left\langle\rho_{*}\left(\hat{b}_{i}^{\prime} \cdot \hat{f}_{k l}^{\prime}\right), f_{s t}\right\rangle\right]\right\| \leq\left\|\left[\rho_{*}\left(\hat{f}_{k l}^{\prime}\right)\right]\right\| .
$$

It follows that

$$
\left\|\left[\rho_{*}\left(\hat{b}^{\prime} \cdot \hat{f}_{k l}^{\prime}\right)\right]\right\| \leq\left\|\left[\rho_{*}\left(\hat{f}_{k l}^{\prime}\right)\right]\right\|
$$

and hence $\hat{b}^{\prime} \in M_{c b}^{r}\left(L_{1}(\mathbb{G})\right)_{1}$.

We can analogously prove that the closed unit ball of $M_{n}\left(M_{c b}^{r}\left(L_{1}(\mathbb{G})\right)\right)$ is weak ${ }^{*}$ closed in $M_{n}\left(L_{\infty}\left(\hat{\mathbb{G}}^{\prime}\right)\right)$ for general $n \in \mathbb{N}$. The only thing we need to remark here is that for this purpose, we need a matricial version of $\left[17\right.$, Proposition 4.1]. That is, we need to show that a matrix element $\left[\hat{b}_{i j}^{\prime}\right] \in M_{n}\left(L_{\infty}\left(\hat{\mathbb{G}}^{\prime}\right)\right)$ is contained in the unit ball of $M_{n}\left(M_{c b}^{r}\left(L_{1}(\mathbb{G})\right)\right)$ if and only if

$$
\left\|\left[\rho_{*}\left(\hat{b}_{i j}^{\prime} \cdot \hat{f}_{k l}^{\prime}\right)\right]\right\| \leq\left\|\left[\rho_{*}\left(\hat{f}_{k l}^{\prime}\right)\right]\right\|
$$

for all $\left[\hat{f}_{k l}^{\prime}\right] \in M_{m}\left(L_{1}\left(\hat{\mathbb{G}}^{\prime}\right)\right)(m \in \mathbb{N})$. This can be shown by the same argument as used in the proof of [17, Proposition 4.1]. We omit the details here.

For any $\hat{f}^{\prime} \in L_{1}\left(\hat{\mathbb{G}}^{\prime}\right)$, we may define a linear functional $\alpha_{\hat{f}^{\prime}}$ on $M_{c b}^{r}\left(L_{1}(\mathbb{G})\right)$ via

$$
\left\langle\alpha_{\hat{f}^{\prime}}, \hat{b}^{\prime}\right\rangle=\left\langle\hat{b}^{\prime}, \hat{f}^{\prime}\right\rangle \text {. }
$$

Since $\|\cdot\|_{L_{\infty}\left(\hat{\mathbb{G}}^{\prime}\right)} \leq\|\cdot\|_{M_{c b}^{r}\left(L_{1}(\mathbb{G})\right)}$, we see that $\alpha_{\hat{f}^{\prime}} \in M_{c b}^{r}\left(L_{1}(\mathbb{G})\right)^{*}$ with $\left\|\alpha_{\hat{f}^{\prime}}\right\| \leq\left\|\hat{f}^{\prime}\right\|$. Hence, we obtain an injective contraction

$$
\alpha: L_{1}\left(\hat{\mathbb{G}}^{\prime}\right) \rightarrow M_{c b}^{r}\left(L_{1}(\mathbb{G})\right)^{*} .
$$

The map $\alpha$ is actually completely contractive, since $\rho\left(L_{1}(\mathbb{G})\right)$ is weak ${ }^{*}$-dense in $L_{\infty}\left(\hat{\mathbb{G}}^{\prime}\right)$, and we have $\|\cdot\|_{M_{n}\left(L_{\infty}\left(\hat{\mathbb{G}}^{\prime}\right)\right)} \leq\|\cdot\|_{M_{n}\left(M_{c b}^{r}\left(L_{1}(\mathbb{G})\right)\right)}$ for all $n \in \mathbb{N}$. Thus we can identify $L_{1}\left(\hat{\mathbb{G}}^{\prime}\right)$ with the subspace $\alpha\left(L_{1}\left(\hat{\mathbb{G}}^{\prime}\right)\right)$ of $M_{c b}^{r}\left(L_{1}(\mathbb{G})\right)^{*}$, which yields a new operator space norm on $L_{1}\left(\hat{\mathbb{G}}^{\prime}\right)$. We let

$$
Q^{r}\left(L_{1}(\mathbb{G})\right)=\overline{\alpha\left(L_{1}\left(\hat{\mathbb{G}}^{\prime}\right)\right)}\|\cdot\| M_{c b}^{r}\left(L_{1}(\mathbb{G})\right)^{*} .
$$

Then

$$
\alpha: \hat{f}^{\prime} \in L_{1}\left(\hat{\mathbb{G}}^{\prime}\right) \rightarrow \alpha_{\hat{f}^{\prime}} \in Q^{r}\left(L_{1}(\mathbb{G})\right)
$$


defines an injective complete contraction from $L_{1}\left(\hat{\mathbb{G}}^{\prime}\right)$ into $Q^{r}\left(L_{1}(\mathbb{G})\right)$. Now we are ready to prove the following quantum group analogue of $[19$, Theorem 6.2].

Theorem 3.4. Let $\mathbb{G}$ be a locally compact quantum group. Then the adjoint of the inclusion map $Q^{r}\left(L_{1}(\mathbb{G})\right) \hookrightarrow M_{c b}^{r}\left(L_{1}(\mathbb{G})\right)^{*}$ induces a complete isometry

$$
M_{c b}^{r}\left(L_{1}(\mathbb{G})\right)=Q^{r}\left(L_{1}(\mathbb{G})\right)^{*} .
$$

Proof. For every $\hat{b}^{\prime} \in M_{c b}^{r}\left(L_{1}(\mathbb{G})\right)$, we define a linear functional $F_{\hat{b}^{\prime}}$ on $L_{1}\left(\hat{\mathbb{G}}^{\prime}\right)$ by

$$
\left\langle F_{\hat{b}^{\prime}}, \hat{f}^{\prime}\right\rangle=\left\langle\hat{b}^{\prime}, \hat{f}^{\prime}\right\rangle
$$

The definition of $Q^{r}\left(L_{1}(\mathbb{G})\right)$ yields that

$$
\left|\left\langle F_{\hat{b}^{\prime}}, \hat{f}^{\prime}\right\rangle\right|=\left|\left\langle\hat{b}^{\prime}, \hat{f}^{\prime}\right\rangle\right| \leq\left\|\hat{b}^{\prime}\right\|_{M_{c b}^{r}\left(L_{1}(\mathbb{G})\right)}\left\|\hat{f}^{\prime}\right\|_{Q^{r}\left(L_{1}(\mathbb{G})\right)}
$$

for all $\hat{f}^{\prime} \in L_{1}\left(\hat{\mathbb{G}}^{\prime}\right)$. Thus $F_{\hat{b}^{\prime}}$ extends to a bounded linear functional on $Q^{r}\left(L_{1}(\mathbb{G})\right)$, which is still denoted by $F_{\hat{b}^{\prime}}$, such that $\left\|F_{\hat{b}^{\prime}}\right\| \leq\left\|\hat{b}^{\prime}\right\|_{M_{c b}^{r}\left(L_{1}(\mathbb{G})\right)}$. Analogously, we see that $\left\|\left[F_{\hat{b}_{i j}^{\prime}}\right]\right\| \leq\left\|\left[\hat{b}_{i j}^{\prime}\right]\right\|_{M_{c b}^{r}\left(L_{1}(\mathbb{G})\right)}$, whence $F: M_{c b}^{r}\left(L_{1}(\mathbb{G})\right) \rightarrow Q^{r}\left(L_{1}(\mathbb{G})\right)^{*}$ is a complete contraction.

On the other hand, suppose that $f \in Q^{r}\left(L_{1}(\mathbb{G})\right)^{*}$ with $\|f\|=1$. Since

$$
\left|\left\langle f, \hat{f}^{\prime}\right\rangle\right| \leq\left\|\hat{f}^{\prime}\right\|_{Q^{r}\left(L_{1}(\mathbb{G})\right)} \leq\left\|\hat{f}^{\prime}\right\|
$$

for all $\hat{f}^{\prime} \in L_{1}\left(\hat{\mathbb{G}}^{\prime}\right)$, we have $\hat{b}_{f}^{\prime}=\left.f\right|_{L_{1}\left(\hat{\mathbb{G}}^{\prime}\right)} \in L_{1}\left(\hat{\mathbb{G}}^{\prime}\right)^{*}=L_{\infty}\left(\hat{\mathbb{G}}^{\prime}\right)$. We claim that $\hat{b}_{f}^{\prime}$ lies in the closed unit ball of $M_{c b}^{r}\left(L_{1}(\mathbb{G})\right)$. Assume that this were false. Then, in view of Proposition 3.3, the Hahn-Banach theorem entails that there is $\hat{f}^{\prime} \in L_{1}\left(\hat{\mathbb{G}}^{\prime}\right)$ such that

$$
\left|\left\langle\hat{b}^{\prime}, \hat{f}^{\prime}\right\rangle\right| \leq 1
$$

for all $\hat{b}^{\prime} \in M_{c b}^{r}\left(L_{1}(\mathbb{G})\right)_{1}$, while

$$
\left\langle\hat{b}_{f}^{\prime}, \hat{f}^{\prime}\right\rangle>1
$$

By (3.7), we have $\left\|\hat{f}^{\prime}\right\|_{Q^{r}\left(L_{1}(\mathbb{G})\right)} \leq 1$, whence

$$
\left|\left\langle\hat{b}_{f}^{\prime}, \hat{f}^{\prime}\right\rangle\right|=\left|\left\langle f, \hat{f}^{\prime}\right\rangle\right| \leq\|f\|\left\|\hat{f}^{\prime}\right\|_{Q^{r}\left(L_{1}(\mathbb{G})\right)} \leq 1,
$$

which contradicts (3.8). Therefore, $\hat{b}_{f}^{\prime} \in M_{c b}^{r}\left(L_{1}(\mathbb{G})\right)_{1}$ as claimed. Since $F_{\hat{b}_{f}^{\prime}}=f$, this shows that $F$ is surjective and $\left\|F_{\hat{b}_{f}^{\prime}}\right\|=\left\|\hat{b}_{f}^{\prime}\right\|_{M_{c b}^{r}\left(L_{1}(\mathbb{G})\right)}$. Thus, $F$ is an isometric isomorphism from $M_{c b}^{r}\left(L_{1}(\mathbb{G})\right)$ onto $Q^{r}\left(L_{1}(\mathbb{G})\right)^{*}$. A corresponding matricial argument shows that $F$ is in fact a complete isometry.

As $M_{c b}^{r}\left(L_{1}(\mathbb{G})\right)$ is a completely contractive Banach algebra, there is a canonical $M_{c b}^{r}\left(L_{1}(\mathbb{G})\right.$ )-bimodule structure on $M_{c b}^{r}\left(L_{1}(\mathbb{G})\right)^{*}$. In particular, for $\omega \in Q^{r}\left(L_{1}(\mathbb{G})\right)$ and $\hat{a}^{\prime} \in M_{c b}^{r}\left(L_{1}(\mathbb{G})\right)$, we have the bounded linear functionals $\hat{a}^{\prime} \cdot \omega$ and $\omega \cdot \hat{a}^{\prime}$ on $M_{c b}^{r}\left(L_{1}(\mathbb{G})\right)$ via

$$
\left\langle\hat{b}^{\prime}, \hat{a}^{\prime} \cdot \omega\right\rangle=\left\langle\hat{b}^{\prime} \hat{a}^{\prime}, \omega\right\rangle \text { and }\left\langle\hat{b}^{\prime}, \omega \cdot \hat{a}^{\prime}\right\rangle=\left\langle\hat{a}^{\prime} \hat{b}^{\prime}, \omega\right\rangle \quad\left(\hat{b}^{\prime} \in M_{c b}^{r}\left(L_{1}(\mathbb{G})\right)\right)
$$


which induce the complete contractions

$$
c_{l}: M_{c b}^{r}\left(L_{1}(\mathbb{G})\right) \hat{\otimes} Q^{r}\left(L_{1}(\mathbb{G})\right) \rightarrow M_{c b}^{r}\left(L_{1}(\mathbb{G})\right)^{*} \text { and } c_{r}: Q^{r}\left(L_{1}(\mathbb{G})\right) \hat{\otimes} M_{c b}^{r}\left(L_{1}(\mathbb{G})\right) \rightarrow M_{c b}^{r}\left(L_{1}(\mathbb{G})\right)^{*}
$$

When $\omega \in L_{1}\left(\hat{\mathbb{G}}^{\prime}\right)$, viewing $\hat{a}^{\prime} \in M_{c b}^{r}\left(L_{1}(\mathbb{G})\right)$ in $L_{\infty}\left(\hat{\mathbb{G}}^{\prime}\right)$, we see that $\hat{a}^{\prime} \cdot \omega$ describes exactly the canonical left action of $L_{\infty}\left(\hat{\mathbb{G}}^{\prime}\right)$ on $L_{1}\left(\hat{\mathbb{G}}^{\prime}\right)$, and hence we have $\hat{a}^{\prime} \cdot \omega \in L_{1}\left(\hat{\mathbb{G}}^{\prime}\right)$. Since $L_{1}\left(\hat{\mathbb{G}}^{\prime}\right)$ is norm dense in $Q^{r}\left(L_{1}(\mathbb{G})\right)$, we conclude that the ranges of the complete contractions $c_{l}$ and $c_{r}$ are contained in $Q^{r}\left(L_{1}(\mathbb{G})\right)$, and thus $Q^{r}\left(L_{1}(\mathbb{G})\right)$ is an operator $M_{c b}^{r}\left(L_{1}(\mathbb{G})\right)$-bimodule. Therefore, we have the following quantum group version of $[19$, Proposition 6.3].

Proposition 3.5. Let $\mathbb{G}$ be a locally compact quantum group. Then $Q^{r}\left(L_{1}(\mathbb{G})\right)$ is an operator $M_{c b}^{r}\left(L_{1}(\mathbb{G})\right)$ bimodule, and $M_{c b}^{r}\left(L_{1}(\mathbb{G})\right)$ is a completely contractive dual Banach algebra.

Identifying $L_{1}(\mathbb{G})$ with the subalgebra $\rho\left(L_{1}(\mathbb{G})\right)$ of $M_{c b}^{r}\left(L_{1}(\mathbb{G})\right)$, we obtain an $L_{1}(\mathbb{G})$-bimodule action on $Q^{r}\left(L_{1}(\mathbb{G})\right)$ given by

$$
f \cdot \omega=\rho(f) \cdot \omega \text { and } \omega \cdot f=\omega \cdot \rho(f)
$$

for $f \in L_{1}(\mathbb{G})$ and $\omega \in Q^{r}\left(L_{1}(\mathbb{G})\right)$. Recall that $\rho\left(L_{1}(\mathbb{G})\right)$ is weak ${ }^{*}$ dense in $L_{\infty}\left(\hat{\mathbb{G}}^{\prime}\right)$. We can thus conclude this section with the following analogue of Proposition 2.2. See Section 4 for further discussions on the relation between $Q^{r}\left(L_{1}(\mathbb{G})\right)$ and $C_{0}(\mathbb{G})$.

Proposition 3.6. Let $\mathbb{G}$ be a locally compact quantum group. Then we have

$$
Q^{r}\left(L_{1}(\mathbb{G})\right)=\left\langle L_{1}(\mathbb{G}) \cdot Q^{r}\left(L_{1}(\mathbb{G})\right)\right\rangle=\left\langle Q^{r}\left(L_{1}(\mathbb{G})\right) \cdot L_{1}(\mathbb{G})\right\rangle .
$$

This also implies that

$$
Q^{r}\left(L_{1}(\mathbb{G})\right)=\left\langle M_{c b}^{r}\left(L_{1}(\mathbb{G})\right) \cdot Q^{r}\left(L_{1}(\mathbb{G})\right)\right\rangle=\left\langle Q^{r}\left(L_{1}(\mathbb{G})\right) \cdot M_{c b}^{r}\left(L_{1}(\mathbb{G})\right)\right\rangle .
$$

Therefore, the space $Q^{r}\left(L_{1}(\mathbb{G})\right)$ can be expressed as

$$
Q^{r}\left(L_{1}(\mathbb{G})\right)=\left\langle L_{1}(\mathbb{G}) \cdot L_{1}\left(\hat{G}^{\prime}\right)\right\rangle=\left\langle L_{1}\left(\hat{G}^{\prime}\right) \cdot L_{1}(\mathbb{G})\right\rangle
$$

via the completely contractive injections $\rho: L_{1}(\mathbb{G}) \rightarrow M_{c b}^{r}\left(L_{1}(\mathbb{G})\right)$ and $\alpha: L_{1}\left(\hat{G}^{\prime}\right) \rightarrow Q^{r}\left(L_{1}(\mathbb{G})\right)$.

4. WEAK*-WEAK* CONTINUITY OF THE REPRESENTATION $\Theta^{r}$ OF $M_{c b}^{r}\left(L_{1}(\mathbb{G})\right)$

Let $\mathbb{G}$ be a locally compact quantum group. In this section, we first show that the completely isometric algebra isomorphism $\Theta^{r}$ in $(1.1)$ is weak*-weak* continuous from $M_{c b}^{r}\left(L_{1}(\mathbb{G})\right)$ onto $\mathcal{C B}_{L_{\infty}(\hat{\mathbb{G}})}^{\sigma, L_{\infty}(\mathbb{G})}\left(\mathcal{B}\left(L_{2}(\mathbb{G})\right)\right)$.

Let us recall from operator space theory that the space $\mathcal{C B}^{\sigma}\left(\mathcal{B}\left(L_{2}(\mathbb{G})\right)\right)$ of normal completely bounded maps on $\mathcal{B}\left(L_{2}(\mathbb{G})\right)$ is a dual operator space with $\mathcal{K}\left(L_{2}(\mathbb{G})\right) \hat{\otimes} \mathcal{T}\left(L_{2}(\mathbb{G})\right)$ as its operator predual, i.e., we have the complete isometries

$$
\mathcal{C B}^{\sigma}\left(\mathcal{B}\left(L_{2}(\mathbb{G})\right)\right)=\mathcal{C B}\left(\mathcal{K}\left(L_{2}(\mathbb{G})\right), \mathcal{B}\left(L_{2}(\mathbb{G})\right)\right)=\left(\mathcal{K}\left(L_{2}(\mathbb{G})\right) \hat{\otimes} \mathcal{T}\left(L_{2}(\mathbb{G})\right)\right)^{*}
$$


Note that the operator projective tensor product space $\mathcal{K}\left(L_{2}(\mathbb{G})\right) \hat{\otimes} \mathcal{T}\left(L_{2}(\mathbb{G})\right)$ can be completely isometrically identified with the Haagerup tensor product space $\mathcal{T}\left(L_{2}(\mathbb{G})\right) \otimes^{h} \mathcal{T}\left(L_{2}(\mathbb{G})\right)$ via the identification

$$
x_{\xi, \eta} \otimes \omega_{\xi^{\prime}, \eta^{\prime}}=\omega_{\xi, \eta^{\prime}} \otimes \omega_{\xi^{\prime}, \eta},
$$

where $x_{\xi, \eta}$ is the rank one operator given by $x_{\xi, \eta}(\zeta)=\langle\zeta \mid \eta\rangle \xi$, and $\omega_{\xi^{\prime}, \eta^{\prime}}$ is the normal linear functional given by $\omega_{\xi^{\prime}, \eta^{\prime}}(x)=\left\langle x \xi^{\prime} \mid \eta^{\prime}\right\rangle$. Thus we can also write

$$
\mathcal{C B}^{\sigma}\left(\mathcal{B}\left(L_{2}(\mathbb{G})\right)\right)=\left(\mathcal{T}\left(L_{2}(\mathbb{G})\right) \otimes^{h} \mathcal{T}\left(L_{2}(\mathbb{G})\right)\right)^{*}=\mathcal{B}\left(L_{2}(\mathbb{G})\right) \otimes^{e h} \mathcal{B}\left(L_{2}(\mathbb{G})\right),
$$

where $\otimes^{e h}$ is the extended Haagerup tensor product (cf. [11], [6], [4], and [7]). Under these identifications, a map $\Phi$ on $\mathcal{B}\left(L_{2}(\mathbb{G})\right)$ is contained in $\mathcal{C B}^{\sigma}\left(\mathcal{B}\left(L_{2}(\mathbb{G})\right)\right)$ if and only if there exist two nets $\left\{a_{i}\right\}$ and $\left\{b_{i}\right\}$ in $\mathcal{B}\left(L_{2}(\mathbb{G})\right)$ such that $\sup \left\{\left\|a_{i}\right\|\right\} \sup \left\{\left\|b_{i}\right\|\right\}=\|\Phi\|_{c b}<\infty$ and

$$
\left\langle\Phi\left(x_{\xi, \eta}\right), \omega_{\xi^{\prime}, \eta^{\prime}}\right\rangle=\sum_{i}\left\langle a_{i} x_{\xi, \eta} b_{i}, \omega_{\xi^{\prime}, \eta^{\prime}}\right\rangle=\sum_{i}\left\langle a_{i} \xi \mid \eta^{\prime}\right\rangle\left\langle b_{i} \xi^{\prime} \mid \eta\right\rangle=\left\langle\Phi, \omega_{\xi, \eta^{\prime}} \otimes \omega_{\xi^{\prime}, \eta}\right\rangle
$$

for all $\xi, \eta, \xi^{\prime}, \eta^{\prime} \in L_{2}(\mathbb{G})$.

Considering the complete quotient

$$
\pi_{\hat{\mathbb{G}}^{\prime}}: \omega \in \mathcal{T}\left(L_{2}(\mathbb{G})\right) \rightarrow \hat{f}^{\prime}=\left.\omega\right|_{L_{\infty}\left(\hat{\mathbb{G}}^{\prime}\right)} \in L_{1}\left(\hat{\mathbb{G}}^{\prime}\right)
$$

via the restriction to $L_{\infty}\left(\hat{\mathbb{G}}^{\prime}\right)$ (see Section 5 for details), we can obtain the complete quotient

$$
\pi_{\hat{\mathbb{G}}^{\prime}} \otimes \pi_{\hat{\mathbb{G}}^{\prime}}: \mathcal{T}\left(L_{2}(\mathbb{G})\right) \otimes^{h} \mathcal{T}\left(L_{2}(\mathbb{G})\right) \rightarrow L_{1}\left(\hat{\mathbb{G}}^{\prime}\right) \otimes^{h} L_{1}\left(\hat{\mathbb{G}}^{\prime}\right) .
$$

The adjoint of $\pi_{\hat{\mathbb{G}}^{\prime}} \otimes \pi_{\hat{\mathbb{G}}^{\prime}}$ is a weak*-weak* continuous completely isometry from $\left(L_{1}\left(\hat{\mathbb{G}}^{\prime}\right) \otimes^{h} L_{1}\left(\hat{\mathbb{G}}^{\prime}\right)\right)^{*}$ into $\mathcal{C B}^{\sigma}\left(\mathcal{B}\left(L_{2}(\mathbb{G})\right)\right)$, whose range space is the weak* closed subspace $\mathcal{C B}_{L_{\infty}(\hat{\mathbb{G}})}^{\sigma}\left(\mathcal{B}\left(L_{2}(\mathbb{G})\right)\right)$ of $\mathcal{C B}^{\sigma}\left(\mathcal{B}\left(L_{2}(\mathbb{G})\right)\right)$ consisting of normal completely bounded $L_{\infty}(\hat{\mathbb{G}})$-bimodule homomorphisms on $\mathcal{B}\left(L_{2}(\mathbb{G})\right)$. This provides us a weak*-homeomorphic completely isometric isomorphism

$$
\mathcal{C B}_{L_{\infty}(\hat{\mathbb{G}})}^{\sigma}\left(\mathcal{B}\left(L_{2}(\mathbb{G})\right)\right) \cong\left(L_{1}\left(\hat{\mathbb{G}}^{\prime}\right) \otimes^{h} L_{1}\left(\hat{\mathbb{G}}^{\prime}\right)\right)^{*}=L_{\infty}\left(\hat{\mathbb{G}}^{\prime}\right) \otimes^{e h} L_{\infty}\left(\hat{\mathbb{G}}^{\prime}\right) .
$$

Then a map $\Phi$ on $\mathcal{B}\left(L_{2}(\mathbb{G})\right)$ is contained in $\mathcal{C B}_{L_{\infty}(\hat{\mathbb{G}})}^{\sigma}\left(\mathcal{B}\left(L_{2}(\mathbb{G})\right)\right)$ if and only if there exist two nets $\left\{\hat{a}_{i}^{\prime}\right\}$ and $\left\{\hat{b}_{i}^{\prime}\right\}$ in $L_{\infty}\left(\hat{\mathbb{G}}^{\prime}\right)$ such that $\Phi(x)=\sum_{i} \hat{a}_{i}^{\prime} x \hat{b}_{i}^{\prime}$ for all $x \in \mathcal{B}\left(L_{2}(\mathbb{G})\right)$. In this case, for given $\hat{f}^{\prime}, \hat{g}^{\prime} \in L_{1}\left(\hat{\mathbb{G}}^{\prime}\right)$, we can find $\xi, \xi^{\prime}, \eta, \eta^{\prime} \in L_{2}(\mathbb{G})$ such that $\hat{f}^{\prime}$ and $\hat{g}^{\prime}$ are the restrictions of $\omega_{\xi, \eta^{\prime}}$ and $\omega_{\xi^{\prime}, \eta}$ to $L_{\infty}\left(\hat{\mathbb{G}}^{\prime}\right)$, respectively. Therefore, we have the duality

$$
\left\langle\Phi, \hat{f}^{\prime} \otimes \hat{g}^{\prime}\right\rangle=\sum_{i} \hat{f}^{\prime}\left(\hat{a}_{i}^{\prime}\right) \hat{g}^{\prime}\left(\hat{b}_{i}^{\prime}\right)=\sum_{i}\left\langle\hat{a}_{i}^{\prime} \xi \mid \eta^{\prime}\right\rangle\left\langle\hat{b}_{i}^{\prime} \xi^{\prime} \mid \eta\right\rangle=\left\langle\Phi\left(x_{\xi, \eta}\right), \omega_{\xi^{\prime}, \eta^{\prime}}\right\rangle
$$

It is also easy to see that $\mathcal{C B}_{L_{\infty}(\widehat{\mathbb{G}})}^{\sigma, L_{\infty}(\mathbb{G})}\left(\mathcal{B}\left(L_{2}(\mathbb{G})\right)\right.$ ) is a weak* closed subspace of $\mathcal{C B}_{L_{\infty}(\hat{\mathbb{G}})}^{\sigma}\left(\mathcal{B}\left(L_{2}(\mathbb{G})\right)\right.$ ) (and hence of $\left.\mathcal{C B}^{\sigma}\left(\mathcal{B}\left(L_{2}(\mathbb{G})\right)\right)\right)$. Then it is a dual operator space and its operator predual is a complete quotient 
of $L_{1}(\hat{\mathbb{G}}) \otimes^{h} L_{1}(\hat{\mathbb{G}})$ (and hence of $\mathcal{T}\left(\mathcal{B}\left(L_{2}(\mathbb{G})\right)\right) \otimes^{h} \mathcal{T}\left(\mathcal{B}\left(L_{2}(\mathbb{G})\right)\right)$ ). Let us still use $\Theta^{r}$ for the completely isometric inclusion

$$
\Theta^{r}: M_{c b}^{r}\left(L_{1}(\mathbb{G})\right) \hookrightarrow \mathcal{C} \mathcal{B}_{L_{\infty}(\hat{\mathbb{G}})}^{\sigma}\left(\mathcal{B}\left(L_{2}(\mathbb{G})\right)\right) \cong L_{\infty}\left(\hat{\mathbb{G}}^{\prime}\right) \otimes^{e h} L_{\infty}\left(\hat{\mathbb{G}}^{\prime}\right) .
$$

Then the restriction $\pi_{Q}^{r}=\left.\left(\Theta^{r}\right)^{*}\right|_{L_{1}\left(\hat{\mathbb{G}}^{\prime}\right) \otimes^{h} L_{1}\left(\hat{\mathbb{G}}^{\prime}\right)}$ of the adjoint map $\left(\Theta^{r}\right)^{*}$ defines a complete contraction from $L_{1}\left(\hat{\mathbb{G}}^{\prime}\right) \otimes^{h} L_{1}\left(\hat{\mathbb{G}}^{\prime}\right)$ into $M_{c b}^{r}\left(L_{1}(\mathbb{G})\right)^{*}$.

Theorem 4.1. Let $\mathbb{G}$ be a locally compact quantum group. Then the induced map $\pi_{Q}^{r}$ is a complete quotient from $L_{1}\left(\hat{\mathbb{G}}^{\prime}\right) \otimes^{h} L_{1}\left(\hat{\mathbb{G}}^{\prime}\right)$ onto $Q^{r}\left(L_{1}(\mathbb{G})\right)$ so that $\left(\pi_{Q}^{r}\right)^{*}=\Theta^{r}$. Therefore,

$$
\Theta^{r}: M_{c b}^{r}\left(L_{1}(\mathbb{G})\right) \cong \mathcal{C B}_{L_{\infty}(\hat{\mathbb{G}})}^{\sigma, L_{\infty}(\mathbb{G})}\left(\mathcal{B}\left(L_{2}(\mathbb{G})\right)\right)
$$

is a weak ${ }^{*}$-homeomorphic completely isometric algebra isomorphism.

Proof. The theorem has been proved in [30, Proposition 4.4 and Proposition 4.8] for the case where $\mathbb{G}$ is commutative or co-commutative. For general $\mathbb{G}$, we need to consider the antipode $\hat{S}^{\prime}$ of $\hat{\mathbb{G}}^{\prime}$, which, in general, is an unbounded operator on $L_{\infty}\left(\hat{\mathbb{G}}^{\prime}\right)$. Recall that the domain $D\left(\hat{S}^{\prime}\right)$ of $\hat{S}^{\prime}$ is a dense subspace of $L_{\infty}\left(\hat{\mathbb{G}}^{\prime}\right)$, which contains all $(\iota \otimes f)\left(V^{*}\right)=(f \otimes \iota)(\hat{V})$ with $f \in L_{1}(\mathbb{G})$, and we have

$$
\hat{S}^{\prime}\left((\iota \otimes f)\left(V^{*}\right)\right)=\hat{S}^{\prime}((f \otimes \iota)(\hat{V}))=(f \otimes \iota)\left(\hat{V}^{*}\right)=(\iota \otimes f)(V),
$$

where $\hat{V}=\Sigma V^{*} \Sigma$. It is seen that, for $\hat{f}^{\prime} \in L_{1}\left(\hat{\mathbb{G}}^{\prime}\right)$, the map $\hat{f}^{\prime} \circ \hat{S}^{\prime}$ is not necessarily contained in $L_{1}\left(\hat{\mathbb{G}}^{\prime}\right)$. Thus we need to consider a relatively smaller space

$L_{1, *}\left(\hat{\mathbb{G}}^{\prime}\right)=\left\{\hat{f}^{\prime} \in L_{1}\left(\hat{\mathbb{G}}^{\prime}\right):\right.$ there exists $\hat{g}^{\prime} \in L_{1}\left(\hat{\mathbb{G}}^{\prime}\right)$ such that $\hat{g}^{\prime}\left(\hat{x}^{\prime}\right)=\hat{f}^{\prime *} \circ \hat{S}^{\prime}\left(\hat{x}^{\prime}\right)$ for all $\left.\hat{x}^{\prime} \in D\left(\hat{S}^{\prime}\right)\right\}$, where $\hat{f}^{\prime *}\left(\hat{x}^{\prime}\right)=\overline{\left\langle\hat{f}^{\prime}, \hat{x}^{\prime *}\right\rangle}\left(\hat{x}^{\prime} \in L_{\infty}\left(\hat{\mathbb{G}}^{\prime}\right)\right)$. It is known by [39, §1.13] that $L_{1, *}\left(\hat{\mathbb{G}}^{\prime}\right)$ is a dense subalgebra of $L_{1}\left(\hat{\mathbb{G}}^{\prime}\right)$. It is worthy to note, though it is not needed here, that there is an involution on $L_{1, *}\left(\hat{\mathbb{G}}^{\prime}\right)$ given by $\hat{f}^{\prime o}=\hat{f}^{\prime *} \circ \hat{S}^{\prime}$ such that $L_{1, *}\left(\hat{\mathbb{G}}^{\prime}\right)$ is an involutive Banach algebra with the norm given by

$$
\left\|\hat{f}^{\prime}\right\|_{L_{1, *}\left(\hat{\mathbb{G}}^{\prime}\right)}=\max \left\{\left\|\hat{f}^{\prime}\right\|,\left\|\hat{f}^{\prime o}\right\|\right\} .
$$

Let $f \in L_{1}(\mathbb{G})$. Then $\rho(f) \in M_{c b}^{r}\left(L_{1}(\mathbb{G})\right)$, and we have

$$
\Theta^{r}(\rho(f))(\cdot)=\Theta_{0}^{r}(f)(\cdot)=(\iota \otimes f)\left(V(\cdot \otimes 1) V^{*}\right) \in \mathcal{C B}_{L_{\infty}(\hat{\mathbb{G}})}^{\sigma}\left(\mathcal{B}\left(L_{2}(\mathbb{G})\right)\right) \cong L_{\infty}\left(\hat{\mathbb{G}}^{\prime}\right) \otimes^{e h} L_{\infty}\left(\hat{\mathbb{G}}^{\prime}\right) .
$$

It follows from the duality $(4.2)$ that for all $\hat{f}^{\prime} \in L_{1}\left(\hat{\mathbb{G}}^{\prime}\right)$ and $\hat{g}^{\prime} \in L_{1, *}\left(\hat{\mathbb{G}}^{\prime}\right)$, we have

$$
\begin{aligned}
\left\langle\pi_{Q}^{r}\left(\hat{f}^{\prime} \otimes \hat{g}^{\prime}\right), \rho(f)\right\rangle & =\left\langle\hat{f}^{\prime} \otimes \hat{g}^{\prime}, \Theta^{r}(\rho(f))\right\rangle=\left\langle\left(\hat{f}^{\prime} \otimes \iota\right)(V)\left(\hat{g}^{\prime} \otimes \iota\right)\left(V^{*}\right), f\right\rangle \\
& =\left\langle\left(\hat{f}^{\prime} \otimes\left(\hat{g}^{\prime *} \circ \hat{S}^{\prime}\right)^{*} \otimes \iota\right)\left(V_{13} V_{23}\right), f\right\rangle=\left\langle\left(\hat{f}^{\prime} \otimes\left(\hat{g}^{\prime *} \circ \hat{S}^{\prime}\right)^{*} \otimes \iota\right)\left(V_{12}^{*} V_{23} V_{12}\right), f\right\rangle \\
& =\left\langle\hat{f}^{\prime} \otimes\left(\hat{g}^{\prime *} \circ \hat{S}^{\prime}\right)^{*}, V^{*}(1 \otimes \rho(f)) V\right\rangle=\left\langle\left(\hat{g}^{\prime *} \circ \hat{S}^{\prime}\right)^{*} \otimes \hat{f}^{\prime}, \hat{V}(\rho(f) \otimes 1) \hat{V}^{*}\right\rangle \\
& =\left\langle\left(\hat{g}^{*} \circ \hat{S}^{\prime}\right)^{*} \star \hat{f}^{\prime}, \rho(f)\right\rangle,
\end{aligned}
$$


where we use in the second equality the fact that

$$
V=\Sigma \hat{V}^{*} \Sigma=\Sigma\left(\iota \otimes \hat{S}^{\prime}\right)(\hat{V}) \Sigma=\left(\hat{S}^{\prime} \otimes \iota\right)\left(V^{*}\right),
$$

and thus

$$
\left(\hat{g}^{\prime} \otimes \iota\right)\left(V^{*}\right)=\left(\hat{g}^{\prime *} \otimes \iota\right)(V)^{*}=\left[\left(\hat{g}^{\prime *} \circ \hat{S}^{\prime} \otimes \iota\right)\left(V^{*}\right)\right]^{*}=\left(\left(\hat{g}^{\prime *} \circ \hat{S}^{\prime}\right)^{*} \otimes \iota\right)(V) .
$$

This shows that

$$
\underline{\pi_{Q}^{r}\left(\hat{f}^{\prime} \otimes \hat{g}^{\prime}\right)=\left(\hat{g}^{\prime *} \circ \hat{S}^{\prime}\right)^{*} \star \hat{f}^{\prime} \in L_{1}\left(\hat{\mathbb{G}}^{\prime}\right) \subseteq Q^{r}\left(L_{1}(\mathbb{G})\right) ? ?}
$$

Therefore, $\pi_{Q}^{r}$ is a complete contraction from $L_{1}\left(\hat{\mathbb{G}}^{\prime}\right) \otimes^{h} L_{1}\left(\hat{\mathbb{G}}^{\prime}\right)$ into $Q^{r}\left(L_{1}(\mathbb{G})\right)$, and hence the adjoint $\operatorname{map}\left(\pi_{Q}^{r}\right)^{*}$ is a weak*-weak ${ }^{*}$ continuous complete contraction from $M_{c b}^{r}\left(L_{1}(\mathbb{G})\right)$ into $L_{\infty}\left(\hat{\mathbb{G}}^{\prime}\right) \otimes^{e h} L_{\infty}\left(\hat{\mathbb{G}}^{\prime}\right)$. Clearly, by definition, we have $\left(\pi_{Q}^{*}\right)^{*}=\Theta^{r}$. It follows that $\pi_{Q}^{r}$ is a complete quotient from $L_{1}\left(\hat{\mathbb{G}}^{\prime}\right) \otimes^{h} L_{1}\left(\hat{\mathbb{G}}^{\prime}\right)$ onto $Q^{r}\left(L_{1}(\mathbb{G})\right)$, and thus $\Theta^{r}$ is a weak*-weak* continuous completely isometric algebra isomorphism from $M_{c b}^{r}\left(L_{1}(\mathbb{G})\right)$ onto $\mathcal{C B}_{L_{\infty}(\hat{\mathbb{G}})}^{\sigma, L_{\infty}(\mathbb{G})}\left(\mathcal{B}\left(L_{2}(\mathbb{G})\right)\right)$.

Remark 4.2. It is shown in [30, Proposition 4.4] that if $G$ is a locally compact group equipped with the left Haar measure $\mu$, then the map

$$
m \circ\left(i d \otimes \kappa_{*}\right): f \otimes g \in L_{1}(G) \otimes^{h} L_{1}(G) \rightarrow f \star \kappa_{*}(g) \in L_{1}(G)
$$

induces a complete quotient map from $L_{1}(G) \otimes^{h} L_{1}(G)$ onto $Q(G)$. If we replace $\mu$ by the right Haar measure $\nu=\mu \circ \kappa_{a}$, then a simple calculation shows that this map is actually the same as the map $\pi_{Q}^{r}$ discussed in Theorem 4.1.

Next, we consider the weak*-weak* continuity of the following canonical embedding of $M(\mathbb{G})$ into $M_{c b}^{r}\left(L_{1}(\mathbb{G})\right)$. Let

$$
\tilde{\rho}: M(\mathbb{G}) \rightarrow M_{c b}^{r}\left(L_{1}(\mathbb{G})\right)
$$

be the composition of the injective completely contractive algebra homomorphism $M(\mathbb{G}) \rightarrow C_{c b}^{r}\left(L_{1}(\mathbb{G})\right)$, $\mu \mapsto m_{\mu}^{r}$ (see Section 3) and the completely isometric algebra isomorphism $C_{c b}^{r}\left(L_{1}(\mathbb{G}) \cong M_{c b}^{r}\left(L_{1}(\mathbb{G})\right)\right.$, $m_{\hat{b}^{\prime}}^{r} \mapsto \hat{b}^{\prime}$ as shown in [17]. Then $\tilde{\rho}: M(\mathbb{G}) \rightarrow M_{c b}^{r}\left(L_{1}(\mathbb{G})\right)$ is an injective completely contractive algebra homomorphism satisfying $\tilde{\rho}(f)=\rho(f)\left(f \in L_{1}(\mathbb{G})\right)$. Therefore, for all $f \in L_{1}(\mathbb{G})$ and $\mu \in M(\mathbb{G})$, we have

$$
\rho(f) \tilde{\rho}(\mu)=\tilde{\rho}(f) \tilde{\rho}(\mu)=\tilde{\rho}(f \star \mu)=\rho(f \star \mu),
$$

and hence $m_{\tilde{\rho}(\mu)}^{r}=m_{\mu}^{r}$.

Note that $\left(\left.\tilde{\rho}\right|_{L_{1}(\mathbb{G})}\right)^{*}: M_{c b}^{r}\left(L_{1}(\mathbb{G})\right)^{*} \rightarrow L_{\infty}(\mathbb{G})$. We let

$$
\tilde{\rho}_{*}=\left.\left(\left.\tilde{\rho}\right|_{L_{1}(\mathbb{G})}\right)^{*}\right|_{Q^{r}\left(L_{1}(\mathbb{G})\right)}: Q^{r}\left(L_{1}(\mathbb{G})\right) \rightarrow L_{\infty}(\mathbb{G}) .
$$

Then $\tilde{\rho}_{*}$ is completely contractive. Moreover, for all $\hat{f}^{\prime} \in L_{1}\left(\hat{\mathbb{G}}^{\prime}\right)$ and $f \in L_{1}(\mathbb{G})$, we have

$$
\left\langle\tilde{\rho}_{*}\left(\hat{f}^{\prime}\right), f\right\rangle=\left\langle\hat{f}^{\prime}, \tilde{\rho}(f)\right\rangle=\left\langle\hat{f}^{\prime}, \rho(f)\right\rangle=\left\langle\rho_{*}\left(\hat{f}^{\prime}\right), f\right\rangle,
$$


and thus $\tilde{\rho}_{*}\left(\hat{f}^{\prime}\right)=\rho_{*}\left(\hat{f}^{\prime}\right) \in C_{0}(\mathbb{G})$. Therefore, we have

$$
\tilde{\rho}_{*}: Q^{r}\left(L_{1}(\mathbb{G})\right) \rightarrow C_{0}(\mathbb{G}) .
$$

Clearly, we have the following two commutative diagrams of complete contractions:

$$
\begin{array}{ccc}
L_{1}\left(\hat{\mathbb{G}}^{\prime}\right) & \stackrel{\rho_{*}}{\longrightarrow} & C_{0}(\mathbb{G}) \\
\alpha \searrow & & \nearrow \tilde{\rho}_{*} \\
& Q^{r}\left(L_{1}(\mathbb{G})\right) & ,
\end{array}
$$

and

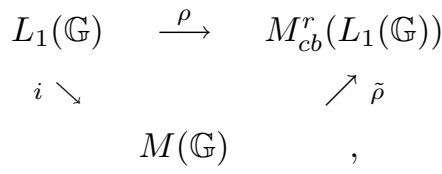

where $i: L_{1}(\mathbb{G}) \rightarrow M(\mathbb{G})$ is the inclusion map.

Recall that $Q^{r}\left(L_{1}(\mathbb{G})\right)$ is an operator $M_{c b}^{r}\left(L_{1}(\mathbb{G})\right)$-bimodule. It is easy to see that the diagrams $(4.3)$ and (4.4) are compatible with the associated canonical bimodule structures. More precisely, for all $\mu \in M(\mathbb{G})$ and $\omega \in Q^{r}\left(L_{1}(\mathbb{G})\right)$, we have

$$
\tilde{\rho}^{*}(\tilde{\rho}(\mu) \cdot \omega)=\mu \star \tilde{\rho}_{*}(\omega) \quad \text { and } \quad \tilde{\rho}^{*}(\omega \cdot \tilde{\rho}(\mu))=\tilde{\rho}_{*}(\omega) \star \mu .
$$

Therefore, not only formally analogous, Proposition 2.2 and Proposition 3.6 are actually related via the intrinsic connection between the $M(\mathbb{G})$-bimodule structure on $C_{0}(\mathbb{G})$ and the $M_{c b}^{r}\left(L_{1}(\mathbb{G})\right)$-bimodule structure on $Q^{r}\left(L_{1}(\mathbb{G})\right)$.

Proposition 4.3. Let $\mathbb{G}$ be a locally compact quantum group. Then $\tilde{\rho}_{*}: Q^{r}\left(L_{1}(\mathbb{G})\right) \rightarrow C_{0}(\mathbb{G})$ is a complete contraction, and

$$
\tilde{\rho}=\left(\tilde{\rho}_{*}\right)^{*}: M(\mathbb{G}) \rightarrow M_{c b}^{r}\left(L_{1}(\mathbb{G})\right)
$$

is a weak*-weak* continuous injective completely contractive algebra homomorphism.

Proof. We only need to show that $\tilde{\rho}=\left(\tilde{\rho}_{*}\right)^{*}$. It is easy to see that $\tilde{\rho}$ and $\left(\tilde{\rho}_{*}\right)^{*}$ agree on $L_{1}(\mathbb{G})$. Let $f \in L_{1}(\mathbb{G})$ and $\mu \in M(\mathbb{G})$. Then

$$
\left(\tilde{\rho}_{*}\right)^{*}(f \star g)=\tilde{\rho}(f \star g)=\tilde{\rho}(f) \tilde{\rho}(g)=\rho(f)\left(\tilde{\rho}_{*}\right)^{*}(g)
$$

for all $g \in L_{1}(\mathbb{G})$. Since $M(\mathbb{G})$ and $M_{c b}^{r}\left(L_{1}(\mathbb{G})\right)$ are dual Banach algebras and $L_{1}(\mathbb{G})$ is $\sigma\left(M(\mathbb{G}), C_{0}(\mathbb{G})\right)$ dense in $M(\mathbb{G})$, we have

$$
\left(\tilde{\rho}_{*}\right)^{*}(f \star \mu)=\rho(f)\left(\tilde{\rho}_{*}\right)^{*}(\mu) .
$$

On the other hand, since $f \star \mu \in L_{1}(\mathbb{G})$, we have

$$
\left(\tilde{\rho}_{*}\right)^{*}(f \star \mu)=\tilde{\rho}(f \star \mu)=\rho(f) \tilde{\rho}(\mu) .
$$


It follows that

$$
\rho(f) \tilde{\rho}(\mu)=\rho(f)\left(\tilde{\rho}_{*}\right)^{*}(\mu) \text { in } M_{c b}^{r}\left(L_{1}(\mathbb{G})\right) \text { and hence in } L_{\infty}\left(\hat{\mathbb{G}}^{\prime}\right) .
$$

Therefore, $\tilde{\rho}(\mu)=\left(\tilde{\rho}_{*}\right)^{*}(\mu)$ for all $\mu \in M(\mathbb{G})$, since $\rho\left(L_{1}(\mathbb{G})\right)$ is weak ${ }^{*}$-dense in $L_{\infty}\left(\hat{\mathbb{G}}^{\prime}\right)$.

The following theorem is the quantum group analogue of Kraus and Ruan [19, Proposition 7.4 and Theorem 7.6]. We are able to provide a much simpler proof here due to the representation theorem in $[17]$.

Theorem 4.4. Let $\mathbb{G}$ be a locally compact quantum group. Then the following are equivalent:

(1) $\mathbb{G}$ is co-amenable;

(2) $\tilde{\rho}: M(\mathbb{G}) \rightarrow M_{c b}^{r}\left(L_{1}(\mathbb{G})\right)$ is a weak*-homeomorphic completely isometric algebra isomorphism;

(3) $\tilde{\rho}_{*}: Q^{r}\left(L_{1}(\mathbb{G})\right) \rightarrow C_{0}(\mathbb{G})$ is a completely isometric linear isomorphism;

$\left(3^{\prime}\right) \tilde{\rho}_{*}: Q^{r}\left(L_{1}(\mathbb{G})\right) \rightarrow C_{0}(\mathbb{G})$ is a bounded linear isomorphism.

In particular, $L_{1}(\mathbb{G})$ is unital, i.e., $\mathbb{G}$ is a discrete quantum group, if and only if we have

$$
L_{1}(\mathbb{G})=M(\mathbb{G})=M_{c b}^{r}\left(L_{1}(\mathbb{G})\right) .
$$

Proof. (1) $\Leftrightarrow(2) \Leftrightarrow(3)$ follows from Proposition 3.1 and Proposition 4.3, and $(3) \Rightarrow\left(3^{\prime}\right)$ is obvious.

Suppose that $\left(3^{\prime}\right)$ holds. Then $\tilde{\rho}_{*}$ is a bounded linear isomorphism from $Q^{r}\left(L_{1}(\mathbb{G})\right)$ onto $C_{0}(\mathbb{G})$, and hence $\tilde{\rho}=\left(\tilde{\rho}_{*}\right)^{*}: M(\mathbb{G}) \rightarrow M_{c b}^{r}\left(L_{1}(\mathbb{G})\right)$ is surjective. Therefore, $M(\mathbb{G})$ is unital, and thus the quantum group $\mathbb{G}$ is co-amenable (cf. [3, Theorem 3.1]).

Using Theorem 4.4 and a similar argument as that given in [19], we can easily obtain the following quantum group analogue of [19, Theorem 7.6]. A direct proof can be found in the recent paper [18] by Kalantar and Neufang.

Theorem 4.5. Let $\mathbb{G}$ be a locally compact quantum group. Then the following are equivalent:

(1) $\hat{\mathbb{G}}^{\prime}$ is co-amenable;

(2) $\|\rho(f)\|=\|f\|$ for all positive $f \in L_{1}(\mathbb{G})$;

(3) There exists $c>0$ such that $\|f\| \leq c\|\rho(f)\|$ for all positive $f \in L_{1}(\mathbb{G})$.

Remark 4.6. (i) For a locally compact group $G$, it is known by Bożejko [5] (for discrete case) and Losert [28] and Ruan [36] (for non-discrete case) that $G$ is amenable if and only if $B(G)=M_{c b}(A(G)$ ). However, it is still open whether the corresponding result holds for general locally compact quantum groups.

(ii) It is seen from Proposition 4.3 that $\tilde{\rho}_{*}: Q^{r}\left(L_{1}(\mathbb{G})\right) \rightarrow C_{0}(\mathbb{G})$ is injective if and only if $\tilde{\rho}(M(\mathbb{G}))$ is weak*-dense in $M_{c b}^{r}\left(L_{1}(\mathbb{G})\right)$, which is true if and only if $\rho\left(L_{1}(\mathbb{G})\right)$ is weak*-dense in $M_{c b}^{r}\left(L_{1}(\mathbb{G})\right)$. By Theorem 4.4, this is the case if $\mathbb{G}$ is co-amenable. It is not clear whether this is true for non-co-amenable quantum groups $\mathbb{G}$. 
(iii) Considering the connection between Propositions 2.2 and 3.6, it might be interesting to see whether there exists a $Q^{r}\left(L_{1}(\mathbb{G})\right)$ action on $M_{c b}^{r}\left(L_{1}(\mathbb{G})\right)$ that is compatible with the canonical $C_{0}(\mathbb{G})$-bimodule structure on $M(\mathbb{G})$ (cf. Proposition 2.1).

Finally, we note that the corresponding results for the completely bounded left centralizer algebra $C_{c b}^{l}\left(L_{1}(\mathbb{G})\right)$ and the completely bounded left multiplier algebra $M_{c b}^{l}\left(L_{1}(\mathbb{G})\right)$ can be obtained via the left regular representation $\lambda$ and the left fundamental unitary $W$. For instance, there is a one-to-one correspondence between $S \in C_{c b}^{l}\left(L_{1}(\mathbb{G})\right)$ and $\hat{a} \in M_{c b}^{l}\left(L_{1}(\mathbb{G})\right)\left(\subseteq L_{\infty}(\hat{\mathbb{G}})\right)$ such that

$$
S=m_{\hat{a}}^{l}: f \in L_{1}(\mathbb{G}) \rightarrow \lambda^{-1}(\hat{a} \lambda(f)) \in L_{1}(\mathbb{G}) .
$$

We can define the space $Q^{l}\left(L_{1}(\mathbb{G})\right)$, show the complete isometry

$$
M_{c b}^{l}\left(L_{1}(\mathbb{G})\right)=Q^{l}\left(L_{1}(\mathbb{G})\right)^{*},
$$

and obtain a weak*-homeomorphic completely isometric anti-algebra isomorphism

$$
\Theta^{l}: M_{c b}^{l}\left(L_{1}(\mathbb{G})\right) \cong \mathcal{C B}_{L_{\infty}\left(\hat{\mathbb{G}}^{\prime}\right)}^{\sigma, L_{\infty}(\mathbb{G})}\left(\mathcal{B}\left(L_{2}(\mathbb{G})\right)\right)
$$

Also, we can prove that $\mathbb{G}$ is co-amenable if and only if $Q^{l}\left(L_{1}(\mathbb{G})\right)=C_{0}(\mathbb{G})$, or equivalently, $M_{c b}^{l}\left(L_{1}(\mathbb{G})\right)=$ $M(\mathbb{G})$, and so on.

It is interesting to remark that, since the two regular representations $\rho$ and $\lambda$ are related by the unitary operator $U=\hat{J} J$ on $L_{2}(\mathbb{G})$, i.e., we have $\rho(\cdot)=U \lambda(\cdot) U^{*}$ (see (2.6)), we can also express $S$ in (4.6) by

$$
S(f)=\rho^{-1}\left(\hat{a}^{\prime} \rho(f)\right)
$$

where $\hat{a}^{\prime}=U \hat{a} U^{*} \in L_{\infty}\left(\hat{\mathbb{G}}^{\prime}\right)$. That is, we can identify $M_{c b}^{l}\left(L_{1}(\mathbb{G})\right)$ with $U\left(M_{c b}^{l}\left(L_{1}(\mathbb{G})\right)\right) U^{*}$, the space of elements $\hat{a}^{\prime}$ in $L_{\infty}\left(\hat{\mathbb{G}}^{\prime}\right)$ such that $\hat{a}^{\prime} \rho(f) \in \rho\left(L_{1}(\mathbb{G})\right)$ and

$$
m_{\hat{a}^{\prime}}^{l}: f \in L_{1}(\mathbb{G}) \rightarrow \rho^{-1}\left(\hat{a}^{\prime} \rho(f)\right) \in L_{1}(\mathbb{G})
$$

is completely bounded.

With this setting, we can obtain the following correspondence between completely bounded double centralizers and completely bounded double multipliers for general locally compact quantum groups.

Theorem 4.7. Let $\mathbb{G}$ be a locally compact quantum group. Then every completely bounded double centralizer $(S, T) \in C_{c b}\left(L_{1}(\mathbb{G})\right)$ is uniquely associated with an operator $\hat{b}^{\prime} \in L_{\infty}\left(\hat{\mathbb{G}}^{\prime}\right)$ such that for all $f \in L_{1}(\mathbb{G})$, we have $\hat{b}^{\prime} \rho(f), \rho(f) \hat{b}^{\prime} \in \rho\left(L_{1}(\mathbb{G})\right)$ and

$$
m_{\hat{b}^{\prime}}^{l}(\cdot)=\rho^{-1}\left(\hat{b}^{\prime} \rho(\cdot)\right) \text { and } m_{\hat{b}^{\prime}}^{r}(\cdot)=\rho^{-1}\left(\rho(\cdot) \hat{b}^{\prime}\right)
$$

are completely bounded maps on $L_{1}(\mathbb{G})$ with $S=m_{\hat{b}^{\prime}}^{l}$ and $T=m_{\hat{b}^{\prime}}^{r}$.

Therefore, we can define the completely bounded double multiplier algebra $M_{c b}\left(L_{1}(\mathbb{G})\right)$ to be the space of all these operators $\hat{b}^{\prime}$ in $L_{\infty}\left(\hat{\mathbb{G}}^{\prime}\right)$, and we can completely identify $C_{c b}\left(L_{1}(\mathbb{G})\right)$ with $M_{c b}\left(L_{1}(\mathbb{G})\right)$. 
Proof. Given any $(S, T) \in C_{c b}\left(L_{1}(\mathbb{G})\right)$, it follows from our discussion above that there exist $\hat{a}^{\prime}$ and $\hat{b}^{\prime}$ in $L_{\infty}\left(\hat{\mathbb{G}}^{\prime}\right)$ such that $\hat{a}^{\prime} \rho(f), \rho(f) \hat{b}^{\prime} \in \rho\left(L_{1}(\mathbb{G})\right)$ and

$$
S(f)=\rho^{-1}\left(\hat{a}^{\prime} \rho(f)\right) \text { and } T(f)=\rho^{-1}\left(\rho(f) \hat{b}^{\prime}\right)
$$

for all $f \in L_{1}(\mathbb{G})$. It suffices to show that $\hat{a}^{\prime}=\hat{b}^{\prime}$. Since $(S, T)$ is a double centralizer on $L_{1}(\mathbb{G})$, we have

$$
f \star \rho^{-1}\left(\hat{a}^{\prime} \rho(g)\right)=f \star S(g)=T(f) \star g=\rho^{-1}\left(\rho(f) \hat{b}^{\prime}\right) \star g
$$

for all $f, g \in L_{1}(\mathbb{G})$. Applying $\rho$ to both sides of the above identity, we obtain

$$
\rho(f) \hat{a}^{\prime} \rho(g)=\rho(f) \hat{b}^{\prime} \rho(g),
$$

and this implies that $\hat{a}^{\prime}=\hat{b}^{\prime}$, since $\rho\left(L_{1}(\mathbb{G})\right)$ is weak ${ }^{*}$-dense in $L_{\infty}\left(\hat{\mathbb{G}}^{\prime}\right)$.

We can analogously define the space $Q\left(L_{1}(\mathbb{G})\right)$ and prove the complete isometry

$$
Q\left(L_{1}(\mathbb{G})\right)^{*}=M_{c b}\left(L_{1}(\mathbb{G})\right)=M_{c b}^{l}\left(L_{1}(\mathbb{G})\right) \cap M_{c b}^{r}\left(L_{1}(\mathbb{G})\right) .
$$

In this case, $Q\left(L_{1}(\mathbb{G})\right)$ is actually completely isometric to $Q^{l}\left(L_{1}(\mathbb{G})\right)+Q^{r}\left(L_{1}(\mathbb{G})\right)$ in the category of operator spaces. We omit the details here.

\section{5. $\operatorname{LUC}(\mathbb{G})$ AND $R U C(\mathbb{G})$}

Let $\mathbb{G}$ be a locally compact quantum group. We recall that the right fundamental unitary $V$ of $\mathbb{G}$ induces a co-associate co-multiplication

$$
\Gamma^{r}: x \in \mathcal{B}\left(L_{2}(\mathbb{G})\right) \rightarrow V(x \otimes 1) V^{*} \in \mathcal{B}\left(L_{2}(\mathbb{G})\right) \bar{\otimes} \mathcal{B}\left(L_{2}(\mathbb{G})\right)
$$

on $\mathcal{B}\left(L_{2}(\mathbb{G})\right)$, and the restriction of $\Gamma^{r}$ to $L_{\infty}(\mathbb{G})$ is equal to the co-multiplication $\Gamma$ on $L_{\infty}(\mathbb{G})$. The pre-adjoint of $\Gamma^{r}$ induces an associative completely contractive multiplication

$$
\triangleright: \omega_{1} \otimes \omega_{2} \in \mathcal{T}\left(L_{2}(\mathbb{G})\right) \hat{\otimes} \mathcal{T}\left(L_{2}(\mathbb{G})\right) \rightarrow \omega_{1} \triangleright \omega_{2}=\left(\omega_{1} \otimes \omega_{2}\right) \circ \Gamma^{r} \in \mathcal{T}\left(L_{2}(\mathbb{G})\right) .
$$

Correspondingly, the left fundamental unitary $W$ of $\mathbb{G}$ induces a co-associate co-multiplication

$$
\Gamma^{l}: x \in \mathcal{B}\left(L_{2}(\mathbb{G})\right) \rightarrow W^{*}(1 \otimes x) W \in \mathcal{B}\left(L_{2}(\mathbb{G})\right) \bar{\otimes} \mathcal{B}\left(L_{2}(\mathbb{G})\right)
$$

on $\mathcal{B}\left(L_{2}(\mathbb{G})\right.$ ), and the restriction of $\Gamma^{l}$ to $L_{\infty}(\mathbb{G})$ is also equal to the co-multiplication $\Gamma$ on $L_{\infty}(\mathbb{G})$. The pre-adjoint of $\Gamma^{l}$ induces another associative completely contractive multiplication

$$
\triangleleft: \omega_{1} \otimes \omega_{2} \in \mathcal{T}\left(L_{2}(\mathbb{G})\right) \hat{\otimes} \mathcal{T}\left(L_{2}(\mathbb{G})\right) \rightarrow \omega_{1} \triangleleft \omega_{2}=\left(\omega_{1} \otimes \omega_{2}\right) \circ \Gamma^{l} \in \mathcal{T}\left(L_{2}(\mathbb{G})\right) .
$$

We remark that in the setting of locally compact groups $G$, the above type of multiplicative structure on $\mathcal{T}\left(L_{2}(G)\right)$ has been studied in [29], [31], [30], and [32]. Let $\hat{J}$ be the conjugate linear isomorphism on $L_{2}(\mathbb{G})$ obtained via the von Neumann algebra $L_{\infty}(\hat{\mathbb{G}})$ with the left Haar weight $\hat{\varphi}$. Then $\tilde{R}(x)=\hat{J} x^{*} \hat{J}$ defines an ${ }^{*}$-anti-automorphism on $\mathcal{B}\left(L_{2}(\mathbb{G})\right)$, which maps $L_{\infty}(\mathbb{G})$ onto $L_{\infty}(\mathbb{G})$ and maps $L_{\infty}(\hat{\mathbb{G}})$ onto 
$L_{\infty}\left(\hat{\mathbb{G}}^{\prime}\right)$. The restriction $R=\left.\tilde{R}\right|_{L_{\infty}(\mathbb{G})}$ is the unitary antipode of $\mathbb{G}$. Using $\tilde{R}$, we obtain a completely isometric involution ${ }^{o}$ on $\mathcal{T}\left(L_{2}(\mathbb{G})\right)$ given by $\omega^{o}=\omega^{*} \circ \tilde{R}\left(\omega \in \mathcal{T}\left(L_{2}(\mathbb{G})\right)\right)$. We can also obtain a completely isometric involution ${ }^{o}$ on $L_{1}(\mathbb{G})$ given by $f^{o}=f^{*} \circ R\left(f \in L_{1}(\mathbb{G})\right)$.

Proposition 5.1. Let $\mathbb{G}$ be a locally compact quantum group. Then the involution ${ }^{\circ}$ on $\mathcal{T}\left(L_{2}(\mathbb{G})\right)$ satisfies

$$
\left(\omega_{1} \triangleright \omega_{2}\right)^{o}=\omega_{2}^{o} \triangleleft \omega_{1}^{o} \quad\left(\omega_{1}, \omega_{2} \in \mathcal{T}\left(L_{2}(\mathbb{G})\right) .\right.
$$

Proof. Recall that the left and right fundamental unitary operators $W$ and $V$ are related by the formula

$$
V=\Sigma(\hat{J} \otimes \hat{J}) W^{*}(\hat{J} \otimes \hat{J}) \Sigma .
$$

Thus, for given $\omega_{1}$ and $\omega_{2}$ in $\mathcal{T}\left(L_{2}(\mathbb{G})\right)$ and $x$ in $\mathcal{B}\left(L_{2}(\mathbb{G})\right)$, we can obtain

$$
\begin{aligned}
\left\langle\left(\omega_{1} \triangleright \omega_{2}\right)^{o}, x\right\rangle & =\overline{\left\langle\omega_{1} \triangleright \omega_{2}, \tilde{R}\left(x^{*}\right)\right\rangle}=\overline{\left\langle\omega_{1} \triangleright \omega_{2}, \hat{J} x \hat{J}\right\rangle}=\overline{\left\langle\omega_{1} \otimes \omega_{2}, V(\hat{J} x \hat{J} \otimes 1) V^{*}\right\rangle} \\
& =\overline{\left\langle\omega_{1} \otimes \omega_{2}, \Sigma(\hat{J} \otimes \hat{J}) W^{*}(1 \otimes x) W(\hat{J} \otimes \hat{J}) \Sigma\right\rangle}=\left\langle\omega_{2}^{o} \triangleleft \omega_{1}^{o}, x\right\rangle .
\end{aligned}
$$

Therefore, we have $\left(\omega_{1} \triangleright \omega_{2}\right)^{o}=\omega_{2}^{o} \triangleleft \omega_{1}^{o}\left(\omega_{1}, \omega_{2} \in \mathcal{T}\left(L_{2}(\mathbb{G})\right)\right)$.

We note that the multiplication $\triangleright$ on $\mathcal{T}\left(L_{2}(\mathbb{G})\right)$ is always left faithful, since $\omega_{1} \triangleright \omega_{2}=0$ for all $\omega_{2} \in \mathcal{T}\left(L_{2}(\mathbb{G})\right)$ implies that $\omega_{1}=0$. However, the multiplication $\triangleright$ is not right faithful on $\mathcal{T}\left(L_{2}(\mathbb{G})\right)$ if $L_{\infty}(\mathbb{G}) \neq \mathcal{B}\left(L_{2}(\mathbb{G})\right)$, or equivalently, if $\mathbb{G}$ is non-trivial. Indeed, since $V \in L_{\infty}\left(\hat{\mathbb{G}}^{\prime}\right) \bar{\otimes} L_{\infty}(\mathbb{G})$, it is easy to see that for $\omega_{1} \in \mathcal{T}\left(L_{2}(\mathbb{G})\right)$ and $x \in \mathcal{B}\left(L_{2}(\mathbb{G})\right)$, we have

$$
\left(\omega_{1} \otimes \iota\right) \circ \Gamma^{r}(x)=\left(\omega_{1} \otimes \iota\right)\left(V(x \otimes 1) V^{*}\right) \in L_{\infty}(\mathbb{G}) .
$$

Therefore, if $\omega_{2}$ is any element in the pre-annihilator $L_{\infty}(\mathbb{G})_{\perp}$ of $L_{\infty}(\mathbb{G})$ in $\mathcal{T}\left(L_{2}(\mathbb{G})\right)$, then $\omega_{1} \triangleright \omega_{2}=0$ for all $\omega_{1} \in \mathcal{T}\left(L_{2}(\mathbb{G})\right)$. Corresponding, the multiplication $\triangleleft$ on $\mathcal{T}\left(L_{2}(\mathbb{G})\right)$ is right faithful, but not left faithful if $\mathbb{G}$ is non-trivial.

The following Lemma shows that we may regard $\left(\mathcal{T}\left(L_{2}(\mathbb{G}), \triangleright\right)\right.$ (respectively, $\left(\mathcal{T}\left(L_{2}(\mathbb{G}), \triangleleft\right)\right)$ as a lifting of $L_{1}(\mathbb{G})$. This phenomenon has been observed in [29], [31], and [30] for $L_{1}(G)$ and $A(G)$ of a locally compact group $G$.

Lemma 5.2. Let $\mathbb{G}$ be a locally compact quantum group. Then the pre-annihilator $L_{\infty}(\mathbb{G})_{\perp}$ of $L_{\infty}(\mathbb{G})$ in $\mathcal{T}\left(L_{2}(\mathbb{G})\right.$ ) is a norm closed two-sided ideal in $\left(\mathcal{T}\left(L_{2}(\mathbb{G})\right), \triangleright\right)$ (respectively, in $\left(\mathcal{T}\left(L_{2}(\mathbb{G})\right), \triangleleft\right)$ ), and the complete quotient map

$$
\omega \in \mathcal{T}\left(L_{2}(\mathbb{G})\right) \rightarrow f=\left.\omega\right|_{L_{\infty}(\mathbb{G})} \in L_{1}(\mathbb{G})
$$

is an involutive completely contractive algebra homomorphism from $\left(\mathcal{T}\left(L_{2}(\mathbb{G})\right), \triangleright\right)$ (respectively, from $\left(\mathcal{T}\left(L_{2}(\mathbb{G})\right), \triangleleft\right)$ ) onto $L_{1}(\mathbb{G})$.

Therefore, we have the completely isometric involutive Banach algebra identification

$$
\left(L_{1}(\mathbb{G}), \star\right) \cong\left(\mathcal{T}\left(L_{2}(\mathbb{G})\right) / L_{\infty}(\mathbb{G})_{\perp}, \triangleright\right)\left(\text { respectively },\left(L_{1}(\mathbb{G}), \star\right) \cong\left(\mathcal{T}\left(L_{2}(\mathbb{G})\right) / L_{\infty}(\mathbb{G})_{\perp}, \triangleleft\right)\right) .
$$


Proof. It suffices to show that for $\omega \in \mathcal{T}\left(L_{2}(\mathbb{G})\right)$ and $\omega^{\prime} \in L_{\infty}(\mathbb{G})_{\perp}$, the products $\omega \triangleright \omega^{\prime}$ and $\omega^{\prime} \triangleright \omega$ are contained in $L_{\infty}(\mathbb{G})_{\perp}$. To see this, let $x$ be an arbitrary element of $L_{\infty}(\mathbb{G})$. Then we have

$$
\left\langle\omega \triangleright \omega^{\prime}, x\right\rangle=\left\langle\omega \otimes \omega^{\prime}, V(x \otimes 1) V^{*}\right\rangle=\left\langle\omega \otimes \omega^{\prime}, \Gamma(x)\right\rangle=0 .
$$

This shows that $\omega \triangleright \omega^{\prime} \in L_{\infty}(\mathbb{G})_{\perp}$. Similarly, we have $\omega^{\prime} \triangleright \omega \in L_{\infty}(\mathbb{G})_{\perp}$. Therefore, $L_{\infty}(\mathbb{G})_{\perp}$ is a norm closed two-sided ideal in $\left(\mathcal{T}\left(L_{2}(\mathbb{G})\right), \triangleright\right)$. We can analogously prove that $L_{\infty}(\mathbb{G})_{\perp}$ is a norm closed two-sided ideal in $\left(\mathcal{T}\left(L_{2}(\mathbb{G})\right), \triangleleft\right)$. The final statement follows from Proposition 5.1 and the fact that $\left.\left(\omega_{1} \triangleright \omega_{2}\right)\right|_{L_{\infty}(\mathbb{G})}=\left.\left(\omega_{1} \triangleleft \omega_{2}\right)\right|_{L_{\infty}(\mathbb{G})}$ for all $\omega_{1}, \omega_{2} \in\left(\mathcal{T}\left(L_{2}(\mathbb{G})\right)\right.$.

The multiplications $\triangleright$ and $\triangleleft$ define on $\mathcal{B}\left(L_{2}(\mathbb{G})\right)$ a completely contractive right $\left(\mathcal{T}\left(L_{2}(\mathbb{G})\right)\right.$, $)$-module structure

$$
(x, \omega) \in \mathcal{B}\left(L_{2}(\mathbb{G})\right) \times \mathcal{T}\left(L_{2}(\mathbb{G})\right) \rightarrow x \triangleright \omega=(\omega \otimes \iota)\left(V(x \otimes 1) V^{*}\right) \in L_{\infty}(\mathbb{G}) \subseteq \mathcal{B}\left(L_{2}(\mathbb{G})\right)
$$

and a completely contractive left $\left(\mathcal{T}\left(L_{2}(\mathbb{G})\right), \triangleleft\right)$-module structure

$$
(\omega, x) \in \mathcal{T}\left(L_{2}(\mathbb{G})\right) \hat{\otimes} \mathcal{B}\left(L_{2}(\mathbb{G})\right) \rightarrow \omega \triangleleft x=(\iota \otimes \omega)\left(W^{*}(1 \otimes x) W\right) \in L_{\infty}(\mathbb{G}) \subseteq \mathcal{B}\left(L_{2}(\mathbb{G})\right) .
$$

In particular, if $x \in L_{\infty}(\mathbb{G})$ and $f=\left.\omega\right|_{L_{\infty}(\mathbb{G})}$ with $\omega \in \mathcal{T}\left(L_{2}(\mathbb{G})\right)$, then we get

$$
x \triangleright \omega=(\omega \otimes \iota) \Gamma(x)=x \star f \text { and } x \triangleleft \omega=(\iota \otimes \omega) \Gamma(x)=f \star x,
$$

where $x \star f$ and $f \star x$ are the natural right and left $L_{1}(\mathbb{G})$-module actions on $L_{\infty}(\mathbb{G})$, respectively.

Recall that the subspaces $\operatorname{LUC}(\mathbb{G})$ and $R U C(\mathbb{G})$ of $L_{\infty}(\mathbb{G})$ are defined by

$$
L U C(\mathbb{G})=\left\langle L_{\infty}(\mathbb{G}) \star L_{1}(\mathbb{G})\right\rangle \quad \text { and } \quad R U C(\mathbb{G})=\left\langle L_{1}(\mathbb{G}) \star L_{\infty}(\mathbb{G})\right\rangle
$$

(cf. [13] and [37]).

Proposition 5.3. Let $\mathbb{G}$ be a locally compact quantum group. Then we have

(1) $\operatorname{LUC}(\mathbb{G})=\left\langle L U C(\mathbb{G}) \star L_{1}(\mathbb{G})\right\rangle=\left\langle\mathcal{B}\left(L_{2}(\mathbb{G})\right) \triangleright \mathcal{T}\left(L_{2}(\mathbb{G})\right)\right\rangle$;

(2) $R U C(\mathbb{G})=\left\langle L_{1}(\mathbb{G}) \star R U C(\mathbb{G})\right\rangle=\left\langle\mathcal{T}\left(L_{2}(\mathbb{G})\right) \triangleleft \mathcal{B}\left(L_{2}(\mathbb{G})\right)\right\rangle$.

Proof. It is clear from the definition that

$$
\left\langle L U C(\mathbb{G}) \star L_{1}(\mathbb{G})\right\rangle \subseteq L U C(\mathbb{G}) \subseteq\left\langle\mathcal{B}\left(L_{2}(\mathbb{G})\right) \triangleright \mathcal{T}\left(L_{2}(\mathbb{G})\right)\right\rangle .
$$

Now given $y \in \mathcal{B}\left(L_{2}(\mathbb{G})\right)$ and $\omega_{1}, \omega_{2} \in \mathcal{T}\left(L_{2}(\mathbb{G})\right)$, we get

$$
x=y \triangleright \omega_{1}=\left(\omega_{1} \otimes \iota\right) V(y \otimes 1) V^{*} \in L_{\infty}(\mathbb{G}),
$$

and thus if let $f_{2}=\left.\omega_{2}\right|_{L_{\infty}(\mathbb{G})} \in L_{1}(\mathbb{G})$, then we have

$$
y \triangleright\left(\omega_{1} \triangleright \omega_{2}\right)=\left(y \triangleright \omega_{1}\right) \triangleright \omega_{2}=x \star f_{2} \in L U C(\mathbb{G}) .
$$


Since the multiplication $\triangleright$ is a complete quotient from $\mathcal{T}\left(L_{2}(\mathbb{G})\right) \hat{\otimes} \mathcal{T}\left(L_{2}(\mathbb{G})\right)$ onto $\mathcal{T}\left(L_{2}(\mathbb{G})\right)$, we have $\left\langle\mathcal{T}\left(L_{2}(\mathbb{G})\right) \triangleright \mathcal{T}\left(L_{2}(\mathbb{G})\right)\right\rangle=\mathcal{T}\left(L_{2}(\mathbb{G})\right)$, and hence we get $\left\langle\mathcal{B}\left(L_{2}(\mathbb{G})\right) \triangleright \mathcal{T}\left(L_{2}(\mathbb{G})\right)\right\rangle \subseteq L U C(\mathbb{G})$. Applying this argument again, we conclude that

$$
\left\langle\mathcal{B}\left(L_{2}(\mathbb{G})\right) \triangleright \mathcal{T}\left(L_{2}(\mathbb{G})\right)\right\rangle \subseteq\left\langle L U C(\mathbb{G}) \star L_{1}(\mathbb{G})\right\rangle .
$$

This proves (1). The proof for (2) is similar.

Proposition 5.4. Let $\mathbb{G}$ be a locally compact quantum group. Then the following are equivalent:

(1) $\mathbb{G}$ is co-amenable;

(2) $\left(\mathcal{T}\left(L_{2}(\mathbb{G})\right), \triangleright\right)$ has a contractive (or bounded) right approximate identity;

(3) $\left(\mathcal{T}\left(L_{2}(\mathbb{G})\right), \triangleleft\right)$ has a contractive (or bounded) left approximate identity.

Proof. We only prove (1) $\Leftrightarrow(2)$. The equivalence (2) $\Leftrightarrow(3)$ follows from Proposition 5.1. Let us first assume that $\left\{f_{\alpha}\right\}$ is a contractive right approximate identity of $L_{1}(\mathbb{G})$. For each $\alpha$, we let $\omega_{\alpha} \in \mathcal{T}\left(L_{2}(\mathbb{G})\right)$ be a contractive normal extension of $f_{\alpha}$. We shall show that $\left\{\omega_{\alpha}\right\}$ is a contractive weak right approximate identity of $\left(\mathcal{T}\left(L_{2}(\mathbb{G})\right), \triangleright\right)$.

Indeed, for given $\omega_{1}, \omega_{2} \in \mathcal{T}\left(L_{2}(\mathbb{G})\right)$, let $f_{i} \in L_{1}(\mathbb{G})$ denote the restriction of $\omega_{i}$ to $L_{\infty}(\mathbb{G})(i=1,2)$. Then for any $x \in \mathcal{B}\left(L_{2}(\mathbb{G})\right)$, we have $x \triangleright \omega_{1} \in L U C(\mathbb{G}) \subseteq L_{\infty}(\mathbb{G})$, and hence

$$
\begin{aligned}
\left\langle\left(\omega_{1} \triangleright \omega_{2}\right) \triangleright \omega_{\alpha}, x\right\rangle & =\left\langle\omega_{2} \triangleright \omega_{\alpha}, x \triangleright \omega_{1}\right\rangle=\left\langle f_{2} \star f_{\alpha}, x \triangleright \omega_{1}\right\rangle \\
& \rightarrow\left\langle f_{2}, x \triangleright \omega_{1}\right\rangle=\left\langle\omega_{2}, x \triangleright \omega_{1}\right\rangle=\left\langle\omega_{1} \triangleright \omega_{2}, x\right\rangle .
\end{aligned}
$$

Since $\left\langle\mathcal{T}\left(L_{2}(\mathbb{G})\right) \triangleright \mathcal{T}\left(L_{2}(\mathbb{G})\right)\right\rangle=\mathcal{T}\left(L_{2}(\mathbb{G})\right)$ and $\left\{\omega_{\alpha}\right\}$ is bounded, for all $\omega \in \mathcal{T}\left(L_{2}(\mathbb{G})\right)$ and $x \in \mathcal{B}\left(L_{2}(\mathbb{G})\right)$, we also have

$$
\left\langle\omega \triangleright \omega_{\alpha}, x\right\rangle \rightarrow\langle\omega, x\rangle .
$$

By a standard convexity argument, we can get a contractive right approximate identity of $\mathcal{T}\left(L_{2}(\mathbb{G})\right)$.

Conversely, it is easy to show that if $\left\{\omega_{\alpha}\right\}$ is a contractive (or bounded) right approximate identity for $\left(\mathcal{T}\left(L_{2}(\mathbb{G})\right), \triangleright\right)$, then $f_{\alpha}=\left.\omega_{\alpha}\right|_{L_{\infty}(\mathbb{G})}$ is a contractive (or bounded) right approximate identity for $L_{1}(\mathbb{G})$.

The corollary below is an immediate consequence of Proposition 5.4 and Cohen's factorization theorem.

Corollary 5.5. If $\mathbb{G}$ is a co-amenable locally compact quantum group, then we have

(1) $\operatorname{LUC}(\mathbb{G})=L U C(\mathbb{G}) \star L_{1}(\mathbb{G})=L_{\infty}(\mathbb{G}) \star L_{1}(\mathbb{G})=\mathcal{B}\left(L_{2}(\mathbb{G})\right) \triangleright \mathcal{T}\left(L_{2}(\mathbb{G})\right)$;

(2) $R U C(\mathbb{G})=L_{1}(\mathbb{G}) \star R U C(\mathbb{G})=L_{1}(\mathbb{G}) \star L_{\infty}(\mathbb{G})=\mathcal{T}\left(L_{2}(\mathbb{G})\right) \triangleleft \mathcal{B}\left(L_{2}(\mathbb{G})\right)$.

By Proposition 2.2, we see that $C_{0}(\mathbb{G}) \subseteq L U C(\mathbb{G})$. It is shown by Runde [37] that $L U C(\mathbb{G})$ is an operator system such that

$$
C_{0}(\mathbb{G}) \subseteq L U C(\mathbb{G}) \subseteq M\left(C_{0}(\mathbb{G})\right) .
$$


Runde further showed that if $\mathbb{G}$ is co-amenable and $C_{0}(\mathbb{G})$ has a bounded approximate identity contained in the centre of $C_{0}(\mathbb{G})$, then $L U C(\mathbb{G})$ is a unital $\mathrm{C}^{*}$-subalgebra of $M\left(C_{0}(\mathbb{G})\right.$ ) (cf. [37, Theorem 5.3]). Moreover, results obtained by Salmi $[38]$ show that $L U C(\mathbb{G})$ is a unital $\mathrm{C}^{*}$-algebra if $\mathbb{G}$ is co-amenable and the right fundamental unitary operator of $\mathbb{G}$ is regular.

Let us recall from Baaj and Skandalis [1] that a fundamental unitary operator $V$ is regular (respectively,



Theorem 5.6. Let $\mathbb{G}$ be a semi-regular locally compact quantum group. Then $L U C(\mathbb{G})$ and $R U C(\mathbb{G})$ are unital $C^{*}$-subalgebras of $M\left(C_{0}(\mathbb{G})\right)$.

Proof. To show that $L U C(\mathbb{G})$ is a unital $\mathrm{C}^{*}$-algebra, we only need to prove that $L U C(\mathbb{G})$ is closed under multiplication. We shall use below the expression $L U C(\mathbb{G})=\left\langle\mathcal{B}\left(L_{2}(\mathbb{G})\right) \triangleright \mathcal{T}\left(L_{2}(\mathbb{G})\right.\right.$ ) (cf. Proposition 5.3).

Suppose that $y_{i} \in \mathcal{B}\left(L_{2}(\mathbb{G})\right)$ and $\omega_{i}=\omega_{\xi_{i}, \eta_{i}} \in \mathcal{T}\left(L_{2}(\mathbb{G})\right)(i=1,2)$. We can write

$$
\omega_{i}(x)=\left\langle x \xi_{i} \mid \eta_{i}\right\rangle=\eta_{i}^{*} x \xi_{i}
$$

for $x \in \mathcal{B}\left(L_{2}(\mathbb{G})\right)$. We want to show that

$$
\left(y_{1} \triangleright \omega_{1}\right)\left(y_{2} \triangleright \omega_{2}\right)=\left(\eta_{1}^{*} \otimes \eta_{2}^{*} \otimes \iota\right) V_{13}\left(y_{1} \otimes 1 \otimes 1\right) V_{13}^{*} V_{23}\left(1 \otimes y_{2} \otimes 1\right) V_{23}^{*}\left(\xi_{1} \otimes \xi_{2} \otimes \iota\right)
$$

is contained in $L U C(\mathbb{G})$. Notice that we can move $\eta_{2}^{*}$ in the 2 nd-leg and $\xi_{1}$ in the 1 st-leg together to the middle (between $V_{13}^{*}$ and $V_{23}$ ) to form the rank one operator $x=x_{\xi_{1}, \eta_{2}}$, and thus we can write (5.2) as

$$
\left(y_{1} \triangleright \omega_{1}\right)\left(y_{2} \triangleright \omega_{2}\right)=\left(\eta_{1}^{*} \otimes \iota\right) V\left(y_{1} \otimes 1\right) V^{*}(x \otimes 1) V\left(y_{2} \otimes 1\right) V^{*}\left(\xi_{2} \otimes \iota\right) .
$$

Since $\mathbb{G}$ is semi-regular, the rank one operator $x$ can be approximated in norm by operators of the form

$$
\sum_{k=1}^{N}\left(\iota \otimes \omega_{\xi_{k}, \eta_{k}}\right)\left(V^{*} \Sigma\right)=\sum_{k=1}^{N}\left(\iota \otimes \eta_{k}^{*}\right) V^{*}\left(\xi_{k} \otimes \iota\right) .
$$

Therefore, we can replace $x$ in $(5.3)$ by operators $\tilde{x}=\left(\iota \otimes \eta^{*}\right) V^{*}(\xi \otimes \iota)$, and it suffices now to show that

$$
\begin{aligned}
& \left(\eta_{1}^{*} \otimes \iota\right) V\left(y_{1} \otimes 1\right) V^{*}(\tilde{x} \otimes 1) V\left(y_{2} \otimes 1\right) V^{*}\left(\xi_{2} \otimes \iota\right) \\
= & \left(\eta_{1}^{*} \otimes \eta^{*} \otimes \iota\right) V_{13}\left(y_{1} \otimes 1 \otimes 1\right) V_{13}^{*} V_{12}^{*} V_{23}\left(1 \otimes y_{2} \otimes 1\right) V_{23}^{*}\left(\xi \otimes \xi_{2} \otimes \iota\right)
\end{aligned}
$$

is contained in $\operatorname{LUC}(\mathbb{G})$. Using the pentagonal relation

$$
V_{13}^{*} V_{12}^{*} V_{23}=V_{23} V_{12}^{*}
$$

we replace $V_{13}^{*} V_{12}^{*} V_{23}$ in (5.4) by $V_{23} V_{12}^{*}$, and we express (5.4) as

$$
\begin{aligned}
& \left(\eta_{1}^{*} \otimes \eta^{*} \otimes \iota\right) V_{13}\left(y_{1} \otimes 1 \otimes 1\right) V_{23} V_{12}^{*}\left(1 \otimes y_{2} \otimes 1\right) V_{23}^{*}\left(\xi \otimes \xi_{2} \otimes \iota\right) \\
= & \left(\eta_{1}^{*} \otimes \eta^{*} \otimes \iota\right) V_{13} V_{23}\left(y_{1} \otimes 1 \otimes 1\right) V_{12}^{*}\left(1 \otimes y_{2} \otimes 1\right) V_{23}^{*}\left(\xi \otimes \xi_{2} \otimes \iota\right) \\
= & \left(\eta_{1}^{*} \otimes \eta^{*} \otimes \iota\right) V_{12}^{*} V_{23} V_{12}\left(y_{1} \otimes 1 \otimes 1\right) V_{12}^{*}\left(1 \otimes y_{2} \otimes 1\right) V_{23}^{*}\left(\xi \otimes \xi_{2} \otimes \iota\right) .
\end{aligned}
$$


Finally, since $\left(\eta_{1}^{*} \otimes \eta^{*}\right) V^{*}$ can be approximated by finite sums $\sum_{j} \tilde{\eta}_{1, j}^{*} \otimes \tilde{\eta}_{2, j}^{*}$ in $L_{2}(\mathbb{G}) \otimes L_{2}(\mathbb{G})$, we can replace $\left(\eta_{1}^{*} \otimes \eta^{*} \otimes \iota\right) V_{12}^{*}$ in $(5.5)$ by $\left(\tilde{\eta}_{1}^{*} \otimes \tilde{\eta}_{2}^{*} \otimes \iota\right)$ and obtain

$\left(\tilde{\eta}_{1}^{*} \otimes \tilde{\eta}_{2}^{*} \otimes \iota\right) V_{23} V_{12}\left(y_{1} \otimes 1 \otimes 1\right) V_{12}^{*}\left(1 \otimes y_{2} \otimes 1\right) V_{23}^{*}\left(\left(\xi \otimes \xi_{2} \otimes \iota\right)\right)=\left(\left(y_{1} \triangleright \omega_{\xi, \tilde{\eta}_{1}}\right) y_{2}\right) \triangleright \omega_{\xi_{2}, \tilde{\eta}_{2}} \in L U C(\mathbb{G})$.

This shows that $L U C(\mathbb{G})$ is closed under multiplication .

We can analogously prove that $R U C(\mathbb{G})$ is a unital $\mathrm{C}^{*}$-algebra by considering the left fundamental unitary operator $W$. We can also show this by using the fact that $R U C(\mathbb{G})$ is a $\mathrm{C}^{*}$-algebra if and only if $L U C(\mathbb{G})$ is a $\mathrm{C}^{*}$-algebra. In fact, for $x \in \mathcal{B}\left(L_{2}(\mathbb{G})\right)$ and $\omega \in \mathcal{T}\left(L_{2}(\mathbb{G})\right)$, by Proposition 5.1 and the argument given in [37], we have

$$
\left[\omega^{\circ} \triangleleft \tilde{R}\left(x^{*}\right)\right]^{*}=\tilde{R}(x \triangleright \omega)=\left(\omega^{*}\right)^{\circ} \triangleleft \tilde{R}(x),
$$

and hence $R$ maps $L U C(\mathbb{G})$ onto $R U C(\mathbb{G})$.

Remark 5.7. We expect that the spaces $\operatorname{LUC}(\mathbb{G})$ and $R U C(\mathbb{G})$ are unital $\mathrm{C}^{*}$-algebras for all locally compact quantum groups $\mathbb{G}$.

\section{6. $L U C(\mathbb{G})^{*}$}

Let $\mathbb{G}$ be a locally compact quantum group. It is known from Banach algebra theory that there exist left and right Arens products $\square$ and $\diamond$ on $L_{\infty}(\mathbb{G})^{*}=L_{1}(\mathbb{G})^{* *}$, which are defined by

$$
\langle\mu \square \nu, x\rangle=\langle\mu, \nu \square x\rangle \text { and }\langle\mu \diamond \nu, x\rangle=\langle\nu, x \diamond \mu\rangle
$$

for all $\mu, \nu \in L_{\infty}(\mathbb{G})^{*}$ and $x \in L_{\infty}(\mathbb{G})$. Here $\nu \square x$ and $x \diamond \mu$ are operators in $L_{\infty}(\mathbb{G})$ defined by

$$
\langle\nu \square x, f\rangle=\langle\nu, x \star f\rangle=\langle\nu,(f \otimes \iota) \Gamma(x)\rangle \text { and }\langle x \diamond \mu, f\rangle=\langle\mu, f \star x\rangle=\langle\mu,(\iota \otimes f) \Gamma(x)\rangle .
$$

Then $\left(L_{\infty}(\mathbb{G})^{*}, \square\right)$ and $\left(L_{\infty}(\mathbb{G})^{*}, \diamond\right)$ are completely contractive Banach algebras. In this section, we shall mainly study the completely contractive Banach algebra structure on $\operatorname{LUC}(\mathbb{G})^{*}$ induced by the left Arens product $\square$. The corresponding results for $R U C(\mathbb{G})^{*}$ via the right Arens product $\diamond$ can be obtained analogously.

Suppose that $\nu \in L U C(\mathbb{G})^{*}$. Then we can define a completely bounded linear map

$$
\nu_{L}: x \in L_{\infty}(\mathbb{G}) \rightarrow \nu \square x \in L_{\infty}(\mathbb{G})
$$

on $L_{\infty}(\mathbb{G})$ with $\left\|\nu_{L}\right\|_{c b} \leq\|\nu\|$. Moreover, the map $\nu_{L}$ is a right $L_{1}(\mathbb{G})$-module homomorphism on $L_{\infty}(\mathbb{G})$, since

$$
\langle\nu \square(x \star f), g\rangle=\langle\nu,(x \star f) \star g\rangle=\langle\nu, x \star(f \star g)\rangle=\langle\nu \square x, f \star g\rangle=\langle(\nu \square x) \star f, g\rangle
$$


for all $x \in L_{\infty}(\mathbb{G})$ and $f, g \in L_{1}(\mathbb{G})$. Therefore, $\nu_{L}$ also maps $L U C(\mathbb{G})$ into $L U C(\mathbb{G})$, i.e., the space $L U C(\mathbb{G})$ is left introverted in $L_{\infty}(\mathbb{G})$. In this situation, the left Arens product $\square$ on $L_{\infty}(\mathbb{G})^{*}$ induces naturally a completely contractive multiplication on $L U C(\mathbb{G})^{*}$, also denoted by $\square$, so that the restriction

$$
\left.\nu \in\left(L_{\infty}(\mathbb{G})^{*}, \square\right) \rightarrow \nu\right|_{L U C(\mathbb{G})} \in\left(L U C(\mathbb{G})^{*}, \square\right)
$$

is a weak ${ }^{*}$-weak* continuous completely contractive Banach algebra quotient from $\left(L_{\infty}(\mathbb{G})^{*}, \square\right)$ onto $\left(\operatorname{LUC}(\mathbb{G})^{*}, \square\right)$.

In the rest of the paper, we will simply use $\operatorname{LUC}(\mathbb{G})^{*}$ to denote the algebra $\left(L U C(\mathbb{G})^{*}, \square\right)$. It is known from [14, Theorem 15] that $L U C(\mathbb{G})^{*}$ is unital (respectively, right unital) if and only if $\mathbb{G}$ is co-amenable.

Recall that $(\mu \otimes \iota) \Gamma(x) \in C_{0}(\mathbb{G})$ and $(\iota \otimes \mu) \Gamma(x) \in C_{0}(\mathbb{G})$ for all $\mu \in M(\mathbb{G})$ and $x \in C_{0}(\mathbb{G})$ (cf. [20, Definition 4.1]). This means that $C_{0}(\mathbb{G})$ is two-sided introverted in $L_{\infty}(\mathbb{G})$. Thus the Arens products $\square$ and $\diamond$ on $L_{\infty}(\mathbb{G})^{*}$ induce two completely contractive multiplications on $C_{0}(\mathbb{G})^{*}$. It is seen in Section 2 that these two Arens products on $C_{0}(\mathbb{G})^{*}$ are the same and both equal to the multiplication $\star$ on $M(\mathbb{G})$. Therefore, the map $L U C(\mathbb{G})^{*} \rightarrow M(\mathbb{G}),\left.\nu \mapsto \nu\right|_{C_{0}(\mathbb{G})}$ is a weak*-weak* continuous completely contractive Banach algebra quotient from $L U C(\mathbb{G})^{*}$ onto $M(\mathbb{G})$.

As mentioned in Section 2, every $\mu \in M(\mathbb{G})$ extends uniquely to a strictly continuous linear functional $\tilde{\mu}$ on $M\left(C_{0}(\mathbb{G})\right)$, which automatically satisfies $\|\tilde{\mu}\|=\|\mu\|$. We let

$$
\pi: M(\mathbb{G}) \rightarrow L U C(\mathbb{G})^{*},\left.\mu \mapsto \tilde{\mu}\right|_{L U C(\mathbb{G})} .
$$

The following two propositions on the map $\pi$ are the quantum group versions of the corresponding results by Ghahramani, Lau and Losert [9] on $L_{1}(G)$, and by Lau and Losert [24] on $A(G)$, noticing that in these two cases, each $\mu \in M(\mathbb{G})$ has a unique norm preserving extension to $\operatorname{LUC}(\mathbb{G})$ (cf. [24, Lemma 1] and [26, Proposition 4.4]). The situation for the $\mathrm{C}^{*}$-algebra $\mathcal{L U C}(\mathbb{G})$ introduced by Salmi is studied in [38].

We point out that the fact that $\pi$ is an isometric algebra homomorphism as shown below is also stated in [34, Lemma 4.1]. However, the proof given in [34] is not appropriately explained, missing the strictly continuous extension property for $\mu$ in $M(\mathbb{G})$.

Proposition 6.1. The map $\pi: M(\mathbb{G}) \rightarrow L U C(\mathbb{G})^{*}$ is a completely isometric algebra homomorphism such that

$$
\operatorname{LUC}(\mathbb{G})^{*}=\pi(M(\mathbb{G})) \oplus C_{0}(\mathbb{G})^{\perp},
$$

where $C_{0}(\mathbb{G})^{\perp}=\left\{\mu \in \operatorname{LUC}(\mathbb{G})^{*}:\left.\mu\right|_{C_{0}(\mathbb{G})}=0\right\}$ is a weak ${ }^{*}$ closed ideal in $\operatorname{LUC}(\mathbb{G})^{*}$.

Therefore, we can completely isometrically and algebraically identify $M(\mathbb{G})$ with the Banach subalgebra $\pi(M(\mathbb{G}))$ of $\operatorname{LUC}(\mathbb{G})^{*}$.

Proof. It is easy to see that $\pi:\left.\mu \in M(\mathbb{G}) \rightarrow \tilde{\mu}\right|_{L U C(\mathbb{G})} \in L U C(\mathbb{G})^{*}$ is a well-defined linear isometry. A natural calculation shows that $\pi$ is a complete isometry. We note that the co-multiplication $\Gamma$ actually 
maps $M\left(C_{0}(\mathbb{G})\right)$ into $M\left(C_{0}(\mathbb{G}) \otimes C_{0}(\mathbb{G})\right)$ and is strictly continuous on the closed unit ball of $M\left(C_{0}(\mathbb{G})\right)$. Then, for any $\nu \in M(\mathbb{G})$, the map

$$
(\widetilde{\iota \otimes \nu}) \circ \Gamma: M\left(C_{0}(\mathbb{G})\right) \rightarrow M\left(C_{0}(\mathbb{G})\right)
$$

is completely bounded and strictly continuous on the closed unit ball of $M\left(C_{0}(\mathbb{G})\right)$. Thus, by the definition of the product $\square$ on $L U C(\mathbb{G})^{*}$, we obtain $\pi(\nu) \square x=\widetilde{(\iota \otimes \nu)} \Gamma(x)(\nu \in M(\mathbb{G}), x \in L U C(\mathbb{G}))$. Therefore, we conclude from $(2.7)$ that

$$
\langle\pi(\mu) \square \pi(\nu), x\rangle=\langle\pi(\mu), \pi(\nu) \square x\rangle=\langle\tilde{\mu},(\widetilde{\iota \otimes \nu}) \Gamma(x)\rangle=\langle\widetilde{\mu \otimes \nu}, \Gamma(x)\rangle=\langle\widetilde{\mu \star \nu}, x\rangle=\langle\pi(\mu \star \nu), x\rangle
$$

for all $\mu, \nu \in M(\mathbb{G})$, and $x \in L U C(\mathbb{G})$. It follows that $\pi: M(\mathbb{G}) \rightarrow L U C(\mathbb{G})^{*}$ is an algebra homomorphism.

It is clear that $C_{0}(\mathbb{G})^{\perp}$ is a weak* closed subspace of $L U C(\mathbb{G})^{*}$, and we can obtain the Banach space decomposition

$$
\operatorname{LUC}(\mathbb{G})^{*}=\pi(M(\mathbb{G})) \oplus C_{0}(\mathbb{G})^{\perp} .
$$

Fix $\nu \in C_{0}(\mathbb{G})^{\perp}, \mu \in L U C(\mathbb{G})^{*}$, and $x \in C_{0}(\mathbb{G})$. Then $\nu \square x=0$, since $x \star f \in C_{0}(\mathbb{G})$ (cf. Proposition 2.2) and thus

$$
\langle\nu \square x, f\rangle=\langle\nu, x \star f\rangle=0
$$

for all $f \in L_{1}(\mathbb{G})$. This shows that $\mu \square \nu \in C_{0}(\mathbb{G})^{\perp}$, and hence $C_{0}(\mathbb{G})^{\perp}$ is a left idea in $L U C(\mathbb{G})^{*}$. To get that $C_{0}(\mathbb{G})^{\perp}$ is a right idea in $L U C(\mathbb{G})^{*}$, we let $\mu_{0}=\left.\mu\right|_{C_{0}(\mathbb{G})} \in M(\mathbb{G})$. Then

$$
\langle\mu \square x, f\rangle=\langle\mu, x \star f\rangle=\left\langle\mu_{0},(f \otimes \iota) \Gamma(x)\right\rangle=\left\langle f,\left(\iota \otimes \mu_{0}\right) \Gamma(x)\right\rangle
$$

for all $f \in L_{1}(\mathbb{G})$. Thus $\mu \square x=\left(\iota \otimes \mu_{0}\right) \Gamma(x) \in C_{0}(\mathbb{G})$. This implies that $\nu \square \mu \in C_{0}(\mathbb{G})^{\perp}$. Therefore, $C_{0}(\mathbb{G})^{\perp}$ is a right idea in $L U C(\mathbb{G})^{*}$.

For every $\mu \in L U C(\mathbb{G})^{*}$, the right multiplication map

$$
\tilde{m}_{\mu}^{r}: \nu \in L U C(\mathbb{G})^{*} \rightarrow \nu \square \mu \in L U C(\mathbb{G})^{*}
$$

is weak*-weak* continuous on $\operatorname{LUC}(\mathbb{G})^{*}$; however, this is not necessarily true for the left multiplication map

$$
\tilde{m}_{\mu}^{l}: \nu \in L U C(\mathbb{G})^{*} \rightarrow \mu \square \nu \in L U C(\mathbb{G})^{*}
$$

We let

$$
\mathcal{Z}_{t}\left(L U C(\mathbb{G})^{*}\right)=\left\{\mu \in L U C(\mathbb{G})^{*}: \tilde{m}_{\mu}^{l} \text { is weak*-weak* continuous on } L U C(\mathbb{G})^{*}\right\}
$$

denote the topological centre of $\operatorname{LUC}(\mathbb{G})^{*}$.

Proposition 6.2. Let $\mathbb{G}$ be a locally compact quantum group. Then we have

$$
M(\mathbb{G}) \subseteq \mathcal{Z}_{t}\left(L U C(\mathbb{G})^{*}\right) \subseteq L U C(\mathbb{G})^{*}
$$


Proof. Let $\mu \in M(\mathbb{G})$. Since $L_{1}(\mathbb{G})$ is a two-sided ideal in $M(\mathbb{G})$, we have $L_{1}(\mathbb{G}) \star \mu \subseteq L_{1}(\mathbb{G})$. By Proposition 2.1, each $f \in L_{1}(\mathbb{G})$ is strictly continuous on $M\left(C_{0}(\mathbb{G})\right)$, and hence $\pi(f)=f$ for all $f \in L_{1}(\mathbb{G})$. We conclude from [14, Corollary 3] that $\mu \in \mathcal{Z}_{t}\left(L U C(\mathbb{G})^{*}\right)$. Therefore, $M(\mathbb{G}) \subseteq \mathcal{Z}_{t}\left(L U C(\mathbb{G})^{*}\right)$.

It is seen that $\operatorname{LUC}(\mathbb{G})^{*}$ is a dual Banach algebra if and only if $\operatorname{LUC}(\mathbb{G})^{*}=\mathcal{Z}_{t}\left(\operatorname{LUC}(\mathbb{G})^{*}\right)$. In the case where $G$ is a locally compact group, this is equivalent to that $L U C(G)^{*}=M(G)$ (since Lau [22] showed that $\mathcal{Z}_{t}\left(L U C(G)^{*}\right)=M(G)$ ), which is true if and only if $G$ is compact. We show in [15] that $L U C(\mathbb{G})^{*}$ being a dual Banach algebra is equivalent to the compactness of $\mathbb{G}$ when either $\mathcal{Z}_{t}\left(L U C(\mathbb{G})^{*}\right)=M(\mathbb{G})$ or $\mathbb{G}$ is amenable with $L_{1}(\mathbb{G})$ separable.

Moreover, we can obtain that $L U C(\mathbb{G})^{*}$ is commutative if and only if $\mathbb{G}$ is co-commutative and compact. In fact, $\operatorname{LUC}(\mathbb{G})^{*}$ is commutative if and only if $L_{1}(\mathbb{G})$ is commutative and $\operatorname{LUC}(\mathbb{G})^{*}=\mathcal{Z}_{t}\left(\operatorname{LUC}(\mathbb{G})^{*}\right)$, which is equivalent to that $L_{1}(\mathbb{G})=A(G)$ for a locally compact group $G$ and $U C B(\hat{G})^{*}$ is commutative; as shown by Lau and Losert in [25, Proposition 5.1], this is exactly the case when $G$ is discrete.

Proposition 6.3. The multiplication $\square$ on $L U C(\mathbb{G})^{*}$ is right faithful, and is left faithful in the following cases:

$(1) \mathbb{G}$ is co-amenable, i.e., $\operatorname{LUC}(\mathbb{G})^{*}$ is unital;

$(2) \mathbb{G}$ is an $S I N$ quantum group, i.e., $L U C(\mathbb{G})=R U C(\mathbb{G})$;

(3) $L_{1}(\mathbb{G})$ is quotient strongly Arens irregular, i.e., $M(\mathbb{G})=\mathcal{Z}_{t}\left(L U C(\mathbb{G})^{*}\right)$.

Proof. Let us first show the right faithfulness of $\square$. Suppose that $\nu \in L U C(\mathbb{G})^{*}$ and $\mu \square \nu=0$ for all $\mu \in L U C(\mathbb{G})^{*}$. Then for all $x \in L U C(\mathbb{G})$ and $f \in L_{1}(\mathbb{G})$, we have $\langle\nu, x \star f\rangle=\langle\nu \square x, f\rangle=\langle f \square \nu, x\rangle=0$. Therefore, we have $\nu=0$ by Proposition 5.3.

We consider now the left faithfulness of $\square$ in the three given cases.

Case (1). Suppose that $\mathbb{G}$ is co-amenable. Then $L U C(\mathbb{G})^{*}$ is unital, and hence $\square$ is faithful.

Case (2). It is easy to see that the multiplication $\square$ on $L U C(\mathbb{G})^{*}$ is left faithful if and only if

$$
\operatorname{LUC}(\mathbb{G})=\left\langle L U C(\mathbb{G})^{*} \square L U C(\mathbb{G})\right\rangle
$$

Suppose that $\mathbb{G}$ is SIN. By Proposition 5.3, we have

$$
\operatorname{LUC}(\mathbb{G})=R U C(\mathbb{G})=\left\langle L_{1}(\mathbb{G}) \star R U C(\mathbb{G})\right\rangle=\left\langle L_{1}(\mathbb{G}) \star L U C(\mathbb{G})\right\rangle
$$

This implies (6.2) and thus the left faithfulness of the multiplication $\square$.

Case (3). Suppose that $\mathcal{Z}_{t}\left(L U C(\mathbb{G})^{*}\right)=M(\mathbb{G})$. Let $\mu \in L U C(\mathbb{G})^{*}$ be such that $\mu \square \nu=0$ for all $\nu \in L U C(\mathbb{G})^{*}$, i.e., $\tilde{m}_{\mu}^{l}=0$ on $L U C(\mathbb{G})^{*}$. Then $\mu \in \mathcal{Z}_{t}\left(L U C(\mathbb{G})^{*}\right)$ and hence $\mu \in M(\mathbb{G})$. Therefore, we have $\mu=0$, since the multiplication on $M(\mathbb{G})$ is faithful (cf. Proposition 2.2). 
Remark 6.4. At this moment, it is not clear whether the multiplication $\square$ on $L U C(\mathbb{G})^{*}$ is always left faithful. We observe that the left faithfulness of $\square$ on $L U C(\mathbb{G})^{*}$ is equivalent to

$$
\mathcal{Z}_{t}\left(L U C(\mathbb{G})^{*}\right) \bigcap\left\langle L_{1}(\mathbb{G}) \star L U C(\mathbb{G})\right\rangle^{\perp}=\{0\} .
$$

Related to (2.5) and Remark 2.3, it is also not clear whether we have

$$
\left\langle L U C(\mathbb{G})^{*} \square L U C(\mathbb{G})^{*}\right\rangle=L U C(\mathbb{G})^{*}
$$

for non-co-amenable locally compact quantum groups $\mathbb{G}$.

As seen in $(6.1)$, each $\nu \in L U C(\mathbb{G})^{*}$ defines a completely bounded right $L_{1}(\mathbb{G})$-module homomorphism $\nu_{L}$ on $L_{\infty}(\mathbb{G})$. Since $\operatorname{LUC}(\mathbb{G})=\left\langle\mathcal{B}\left(L_{2}(\mathbb{G})\right) \triangleright \mathcal{T}\left(L_{2}(\mathbb{G})\right)\right\rangle$, the map

$$
\nu_{L}: x \in \mathcal{B}\left(L_{2}(\mathbb{G})\right) \rightarrow \nu \square x \in \mathcal{B}\left(L_{2}(\mathbb{G})\right)
$$

is also a well-defined completely bounded right $\mathcal{T}\left(L_{2}(\mathbb{G})\right)$-module homomorphism on $\mathcal{B}\left(L_{2}(\mathbb{G})\right)$ with $\left\|\nu_{L}\right\|_{c b} \leq\|\nu\|$, which obviously extends the map given in (6.1). Here we let

$$
\langle\nu \square x, \omega\rangle=\langle\nu, x \triangleright \omega\rangle
$$

for all $x \in \mathcal{B}\left(L_{2}(\mathbb{G})\right)$ and $\omega \in \mathcal{T}\left(L_{2}(\mathbb{G})\right)$.

Let $\mathcal{C B}_{\mathcal{T}\left(L_{2}(\mathbb{G})\right)}\left(\mathcal{B}\left(L_{2}(\mathbb{G})\right)\right)$ (respectively, $\mathcal{C B}_{L_{1}(\mathbb{G})}\left(L_{\infty}(\mathbb{G})\right)$ and $\mathcal{C B}_{L_{1}(\mathbb{G})}(L U C(\mathbb{G}))$ ) denote the space of completely bounded right $\mathcal{T}\left(L_{2}(\mathbb{G})\right.$ )-module homomorphisms on $\mathcal{B}\left(L_{2}(\mathbb{G})\right.$ ) (respectively, the spaces of completely bounded right $L_{1}(\mathbb{G})$-module homomorphisms on $L_{\infty}(\mathbb{G})$ and $L U C(\mathbb{G})$ ). Then the map

$$
\Phi_{L}: \nu \in L U C(\mathbb{G})^{*} \rightarrow \nu_{L} \in \mathcal{C B}\left(\mathcal{B}\left(L_{2}(\mathbb{G})\right)\right)
$$

gives an injective completely contractive algebra homomorphism from $L U C(\mathbb{G})^{*}$ into $\mathcal{C} \mathcal{B}_{\mathcal{T}\left(L_{2}(\mathbb{G})\right)}\left(\mathcal{B}\left(L_{2}(\mathbb{G})\right)\right)$, $\mathcal{C B}_{L_{1}(\mathbb{G})}\left(L_{\infty}(\mathbb{G})\right)$, and $\mathcal{C B}_{L_{1}(\mathbb{G})}(L U C(\mathbb{G}))$, respectively. Clearly, $\Phi_{L}$ is the adjoint map of the completely contractive module product

$$
\mathcal{B}\left(L_{2}(\mathbb{G})\right) \hat{\otimes} \mathcal{T}\left(L_{2}(\mathbb{G})\right) \rightarrow L U C(\mathbb{G}),(x, \omega) \mapsto x \triangleright \omega
$$

(respectively, $\left.L_{\infty}(\mathbb{G}) \hat{\otimes} L_{1}(\mathbb{G}) \rightarrow L U C(\mathbb{G}),(x, f) \mapsto x \star f\right)$. Therefore, $\Phi_{L}$ maps $L U C(\mathbb{G})^{*}$ weak $^{*}$-weak ${ }^{*}$ continuously into $\mathcal{C B}_{\mathcal{T}\left(L_{2}(\mathbb{G})\right)}\left(\mathcal{B}\left(L_{2}(\mathbb{G})\right)\right)$ and $\mathcal{C B}_{L_{1}(\mathbb{G})}\left(L_{\infty}(\mathbb{G})\right)$, respectively.

Proposition 6.5. Let $\mathbb{G}$ be a locally compact quantum group. Then

$$
\Phi_{L}: \nu \in L U C(\mathbb{G})^{*} \rightarrow \nu_{L} \in \mathcal{C B}\left(\mathcal{B}\left(L_{2}(\mathbb{G})\right)\right)
$$

is a weak*-weak* continuous injective completely contractive algebra homomorphism.

Moreover, the quantum group $\mathbb{G}$ is co-amenable if and only if we have one (and hence all) of the following (completely) isometric algebra isomorphisms via $\Phi_{L}$ :

(1) $L U C(\mathbb{G})^{*} \cong \mathcal{C B}_{\mathcal{T}\left(L_{2}(\mathbb{G})\right)}\left(\mathcal{B}\left(L_{2}(\mathbb{G})\right)\right)$; 
(2) $\operatorname{LUC}(\mathbb{G})^{*} \cong \mathcal{C B}_{L_{1}(\mathbb{G})}\left(L_{\infty}(\mathbb{G})\right)$;

(3) $\operatorname{LUC}(\mathbb{G})^{*} \cong \mathcal{C B}_{L_{1}(\mathbb{G})}(L U C(\mathbb{G}))$.

Proof. It is obvious that each of $(1),(2)$, and (3) implies the co-amenability of $\mathbb{G}$, since $L U C(\mathbb{G})^{*}$ is unital in these cases (cf. [14, Theorem 15]).

Conversely, suppose that $\mathbb{G}$ is co-amenable. Then $L_{1}(\mathbb{G})$ and $\left(\mathcal{T}\left(L_{2}(\mathbb{G})\right), \triangleright\right)$ both have contractive right approximate identities (cf. Proposition 5.4). Due to [23, Theorem 5.1], we have the isometric algebra isomorphisms (1), (2), and (3). A standard matricial argument shows that they are actually complete isometries.

Finally, we note that $\Phi_{L}$ also induces an injective completely contractive algebra homomorphism $M(\mathbb{G}) \rightarrow \mathcal{C B}_{L_{1}(\mathbb{G})}\left(C_{0}(\mathbb{G})\right)$, which is precisely corresponding to the left $M(\mathbb{G})$-module action on $C_{0}(\mathbb{G})$, where $\mathcal{C B}_{L_{1}(\mathbb{G})}\left(C_{0}(\mathbb{G})\right)$ is the space of completely bounded right $L_{1}(\mathbb{G})$-module homomorphisms on $C_{0}(\mathbb{G})$.

Under the embedding $\pi: M(\mathbb{G}) \hookrightarrow L U C(\mathbb{G})^{*}$, we can obtain $M(\mathbb{G}) \cong \mathcal{C} \mathcal{B}_{\mathcal{T}\left(L_{2}(\mathbb{G})\right)}^{\sigma}\left(\mathcal{B}\left(L_{2}(\mathbb{G})\right)\right)$ via $\Phi_{L}$ when $\mathbb{G}$ is co-amenable. Therefore, replacing $\operatorname{LUC}(\mathbb{G})^{*}$ by $M(\mathbb{G})$ and combining Theorem 4.4 with Bédos and Tuset [3, Theorem 3.1] and Lau [23, Theorem 5.1], we can show that the co-amenability of $\mathbb{G}$ is also characterized by one (and hence all) of the following (completely) isometric algebra isomorphisms:

$$
M(\mathbb{G}) \cong \mathcal{C B}_{\mathcal{T}\left(L_{2}(\mathbb{G})\right)}^{\sigma}\left(\mathcal{B}\left(L_{2}(\mathbb{G})\right)\right) ; \quad M(\mathbb{G}) \cong \mathcal{C B}_{L_{1}(\mathbb{G})}^{\sigma}\left(L_{\infty}(\mathbb{G})\right) ; \quad M(\mathbb{G}) \cong \mathcal{C} \mathcal{B}_{L_{1}(\mathbb{G})}\left(C_{0}(\mathbb{G})\right)
$$

where the first two isomorphisms are also determined by the map $\tilde{\rho}$ in Proposition 4.3. We omit the details here.

\section{7. $U C Q^{r}\left(L_{1}(\mathbb{G})\right)$ AND $U C Q^{r}\left(L_{1}(\mathbb{G})\right)^{*}$}

As we have discussed in Sections 5 and 6 , given a locally compact quantum group $\mathbb{G}$, there exits a completely contractive multiplication $\triangleright$ on $\mathcal{T}\left(L_{2}(\mathbb{G})\right.$ ) associated with the right fundamental unitary $V$. We shall simply use $\mathcal{T}\left(L_{2}(\mathbb{G})\right)$ to denote the algebra $\left(\mathcal{T}\left(L_{2}(\mathbb{G})\right), \triangleright\right)$. Then $\triangleright$ induces a completely contractive right $\mathcal{T}\left(L_{2}(\mathbb{G})\right)$-module structure

$$
x \triangleright \omega=(\omega \otimes \iota) \Gamma^{r}(x)=(\omega \otimes \iota)\left(V(x \otimes 1) V^{*}\right)
$$

on $\mathcal{B}\left(L_{2}(\mathbb{G})\right)$, and thus we obtain a complete contraction

$$
\tilde{\mathcal{S}}^{r}: x \otimes \omega \in \mathcal{B}\left(L_{2}(\mathbb{G})\right) \hat{\otimes} \mathcal{T}\left(L_{2}(\mathbb{G})\right) \rightarrow x \triangleright \omega=(\omega \otimes \iota) \Gamma^{r}(x) \in L U C(\mathbb{G}) \subseteq L_{\infty}(\mathbb{G}) .
$$

We let $U C Q^{r}\left(L_{1}(\mathbb{G})\right)$ denote the range space of $\tilde{\mathcal{S}}^{r}$, that is equipped with the operator space matrix norm stemming from the quotient space $\mathcal{B}\left(L_{2}(\mathbb{G})\right) \hat{\otimes} \mathcal{T}\left(L_{2}(\mathbb{G})\right) / \operatorname{ker} \tilde{\mathcal{S}}^{r}$. Then we obtain the commutative 
diagram of complete contractions:

$$
\begin{array}{ccc}
\mathcal{B}\left(L_{2}(\mathbb{G})\right) \hat{\otimes} \mathcal{T}\left(L_{2}(\mathbb{G})\right) & \stackrel{\tilde{S}^{r}}{\longrightarrow} & \operatorname{LUC}(\mathbb{G}) \\
& & \nearrow \tilde{\boldsymbol{\tau}}^{r} \\
& \tilde{\pi}^{r} \searrow & ,
\end{array}
$$

where $\tilde{\pi}^{r}$ is the complete quotient map from $\mathcal{B}\left(L_{2}(\mathbb{G})\right) \hat{\otimes} \mathcal{T}\left(L_{2}(\mathbb{G})\right)$ onto $U C Q^{r}\left(L_{1}(\mathbb{G})\right)$, and $\tilde{\iota}^{r}$ is the induced completely contractive injection (or inclusion) from $U C Q^{r}\left(L_{1}(\mathbb{G})\right)$ into $L U C(\mathbb{G})$.

In the following, we shall consider a natural completely contractive Banach algebra structure on the space $U C Q^{r}\left(L_{1}(\mathbb{G})\right)^{*}$. First, we note that for $x \in \mathcal{B}\left(L_{2}(\mathbb{G})\right)$ and $\omega, \omega^{\prime} \in \mathcal{T}\left(L_{2}(\mathbb{G})\right)$, we have

$$
(x \triangleright \omega) \triangleright \omega^{\prime}=x \triangleright\left(\omega \triangleright \omega^{\prime}\right) \in U C Q^{r}\left(L_{1}(\mathbb{G})\right),
$$

and

$$
\|x \triangleright \omega\|_{U C Q^{r}\left(L_{1}(\mathbb{G})\right)} \leq\|x\|_{\mathcal{B}\left(L_{2}(\mathbb{G})\right)}\|\omega\|_{\mathcal{T}\left(L_{2}(\mathbb{G})\right)} .
$$

In particular, we have

$$
\|y \triangleright \omega\|_{U C Q^{r}\left(L_{1}(\mathbb{G})\right)} \leq\|y\|_{U C Q^{r}\left(L_{1}(\mathbb{G})\right)}\|\omega\|_{\mathcal{T}\left(L_{2}(\mathbb{G})\right)}
$$

for all $y \in U C Q^{r}\left(L_{1}(\mathbb{G})\right)$ and $\omega \in \mathcal{T}\left(L_{2}(\mathbb{G})\right)$. Therefore, the right $\mathcal{T}\left(L_{2}(\mathbb{G})\right)$-module action on $\mathcal{B}\left(L_{2}(\mathbb{G})\right)$ induces a right $\mathcal{T}\left(L_{2}(\mathbb{G})\right)$-module structure on $U C Q^{r}\left(L_{1}(\mathbb{G})\right)$.

Next, we note that for $n \in U C Q^{r}\left(L_{1}(\mathbb{G})\right)^{*}$ and $x \in \mathcal{B}\left(L_{2}(\mathbb{G})\right)$, we can define $n \triangleright x \in \mathcal{B}\left(L_{2}(\mathbb{G})\right)$ by

$$
\langle n \triangleright x, \omega\rangle=\langle n, x \triangleright \omega\rangle\left(\omega \in \mathcal{T}\left(L_{2}(\mathbb{G})\right)\right),
$$

and we have

$$
\|n \triangleright x\|_{\mathcal{B}\left(L_{2}(\mathbb{G})\right)} \leq\|n\|\|\omega\|_{\mathcal{T}\left(L_{2}(\mathbb{G})\right)} .
$$

It is easy to see that

$$
n \triangleright(x \triangleright \omega)=(n \triangleright x) \triangleright \omega \in U C Q^{r}\left(L_{1}(\mathbb{G})\right)
$$

for all $n \in U C Q^{r}\left(L_{1}(\mathbb{G})\right)^{*}, x \in \mathcal{B}\left(L_{2}(\mathbb{G})\right)$, and $\omega \in \mathcal{T}\left(L_{2}(\mathbb{G})\right)$. The argument used in the proof of [33, Lemma 2.1] shows that for $n \in U C Q^{r}\left(L_{1}(\mathbb{G})\right)^{*}, x_{1}, \cdots, x_{k} \in \mathcal{B}\left(L_{2}(\mathbb{G})\right)$, and $\omega_{1}, \cdots, \omega_{k} \in \mathcal{T}\left(L_{2}(\mathbb{G})\right)$, we have

$$
\left\|n \triangleright \sum_{i=1}^{k}\left(x_{i} \triangleright \omega_{i}\right)\right\|_{U C Q^{r}\left(L_{1}(\mathbb{G})\right)} \leq\|n\|\left\|\sum_{i=1}^{k}\left(x_{i} \triangleright \omega_{i}\right)\right\|_{U C Q^{r}\left(L_{1}(\mathbb{G})\right)} .
$$

It follows that for all $n \in U C Q^{r}\left(L_{1}(\mathbb{G})\right)^{*}$ and $y \in U C Q^{r}\left(L_{1}(\mathbb{G})\right)$, we have $n \triangleright y \in U C Q^{r}\left(L_{1}(\mathbb{G})\right)$ and

$$
\|n \triangleright y\|_{U C Q^{r}\left(L_{1}(\mathbb{G})\right)} \leq\|n\|\|y\|_{U C Q^{r}\left(L_{1}(\mathbb{G})\right)} .
$$

Finally, we are ready to define a Banach algebra multiplication on $U C Q^{r}\left(L_{1}(\mathbb{G})\right)^{*}$ by letting

$$
\langle m \triangleright n, y\rangle=\langle m, n \triangleright y\rangle \quad\left(m, n \in U C Q^{r}\left(L_{1}(\mathbb{G})\right)^{*}, y \in U C Q^{r}\left(L_{1}(\mathbb{G})\right)\right) .
$$


Then we have $m \triangleright n \in U C Q^{r}\left(L_{1}(\mathbb{G})\right)^{*}$ and

$$
\|m \triangleright n\| \leq\|m\|\|n\|
$$

for all $m, n \in U C Q^{r}\left(L_{1}(\mathbb{G})\right)^{*}$. In the rest of this section, the Banach algebra $\left(U C Q^{r}\left(L_{1}(\mathbb{G})\right)^{*}, \triangleright\right)$ will simply be denoted by $U C Q^{r}\left(L_{1}(\mathbb{G})\right)^{*}$.

Let $\tilde{\Theta}^{r}=\left(\tilde{\pi}^{r}\right)^{*}$ be the adjoint map of $\tilde{\pi}^{r}$. Then $\tilde{\Theta}^{r}: U C Q^{r}\left(L_{1}(\mathbb{G})\right)^{*} \rightarrow \mathcal{C B}\left(\mathcal{B}\left(L_{2}(\mathbb{G})\right)\right)$ is a weak ${ }^{*}$ weak* continuous completely isometric injection. It is easy to see that $\tilde{\Theta}^{r}$ maps $U C Q^{r}\left(L_{1}(\mathbb{G})\right)^{*}$ into $\mathcal{C B}_{\mathcal{T}\left(L_{2}(\mathbb{G})\right)}\left(\mathcal{B}\left(L_{2}(\mathbb{G})\right)\right)$. It is also easy to see that

$$
\tilde{\Theta}^{r}: U C Q^{r}\left(L_{1}(\mathbb{G})\right)^{*} \rightarrow \mathcal{C} \mathcal{B}_{\mathcal{T}\left(L_{2}(\mathbb{G})\right)}\left(\mathcal{B}\left(L_{2}(\mathbb{G})\right)\right)
$$

is an algebra homomorphism. This shows in particular that $U C Q^{r}\left(L_{1}(\mathbb{G})\right)^{*}$ is a completely contractive Banach algebra. Note that $V \in L_{\infty}\left(\hat{\mathbb{G}}^{\prime}\right) \bar{\otimes} L_{\infty}(\mathbb{G})$, and

$$
\left\langle\tilde{\Theta}^{r}(n)(x), \omega\right\rangle=\langle n, x \triangleright \omega\rangle=\left\langle n,(\omega \otimes \iota) V(x \otimes 1) V^{*}\right\rangle
$$

for all $n \in U C Q^{r}\left(L_{1}(\mathbb{G})\right)^{*}, x \in \mathcal{B}\left(L_{2}(\mathbb{G})\right)$, and $\omega \in \mathcal{T}\left(L_{2}(\mathbb{G})\right)$. We can express the above as

$$
\Theta^{r}(n)(x)=n \triangleright x=(\iota \otimes n) V(x \otimes 1) V^{*} .
$$

Thus for all $n \in U C Q^{r}\left(L_{1}(\mathbb{G})\right)^{*}$, the map $\tilde{\Theta}^{r}(n)$ is an $L_{\infty}(\hat{\mathbb{G}})$-bimodule homomorphism on $\mathcal{B}\left(L_{2}(\mathbb{G})\right)$ leaving $L_{\infty}(\mathbb{G})$ invariant. That is, we also have

$$
\tilde{\Theta}^{r}: U C Q^{r}\left(L_{1}(\mathbb{G})\right)^{*} \rightarrow \mathcal{C B}_{L_{\infty}(\hat{\mathbb{G}})}^{L_{\infty}(\mathbb{B})}\left(\mathcal{B}\left(L_{2}(\mathbb{G})\right)\right)
$$

Summarizing the above discussions, we have the following.

Theorem 7.1. Let $\mathbb{G}$ be a locally compact quantum group. Then $U C Q^{r}\left(L_{1}(\mathbb{G})\right)^{*}$ is a completely contractive Banach algebra, and

$$
\tilde{\Theta}^{r}: U C Q^{r}\left(L_{1}(\mathbb{G})\right)^{*} \rightarrow \mathcal{C B}\left(\mathcal{B}\left(L_{2}(\mathbb{G})\right)\right)
$$

is a weak ${ }^{*}$-weak* continuous completely isometric algebra homomorphism satisfying

$$
\tilde{\Theta}^{r}\left(U C Q^{r}\left(L_{1}(\mathbb{G})\right)^{*}\right) \subseteq \mathcal{C B}_{\mathcal{T}\left(L_{2}(\mathbb{G})\right)}\left(\mathcal{B}\left(L_{2}(\mathbb{G})\right)\right) \cap \mathcal{C B}_{L_{\infty}(\hat{\mathbb{G}})}^{L_{\infty}(\mathbb{B})}\left(\mathcal{B}\left(L_{2}(\mathbb{G})\right)\right) .
$$

Since the inclusion map $\tilde{\iota}^{r}$ in $(7.1)$ is a complete contraction from $U C Q^{r}\left(L_{1}(\mathbb{G})\right)$ into $L U C(\mathbb{G})$ with a norm dense range, its adjoint map

$$
\tilde{\Xi}^{r}=\left(\tilde{\iota}^{r}\right)^{*}: L U C(\mathbb{G})^{*} \rightarrow U C Q^{r}\left(L_{1}(\mathbb{G})\right)^{*}
$$

is a weak ${ }^{*}$-weak ${ }^{*}$ continuous injective completely contractive algebra homomorphism with a weak ${ }^{*}$-dense range. Then the composition map

$$
\tilde{\Theta}^{r} \circ \tilde{\Xi}^{r}: L U C(\mathbb{G})^{*} \rightarrow \mathcal{C B}\left(\mathcal{B}\left(L_{2}(\mathbb{G})\right)\right)
$$


is a weak*-weak* injective continuous completely contractive algebra homomorphism, which is actually equal to the map $\Phi_{L}$ in $(6.4)$. In fact, for all $\nu \in L U C(\mathbb{G})^{*}, x \in \mathcal{B}\left(L_{2}(\mathbb{G})\right)$, and $\omega \in \mathcal{T}\left(L_{2}(\mathbb{G})\right)$, we have

$$
\left\langle\Phi_{L}(\nu)(x), \omega\right\rangle=\langle\nu, x \triangleright \omega\rangle=\left\langle\tilde{\Xi}^{r}(\nu), x \triangleright \omega\right\rangle=\left\langle\tilde{\Theta}^{r}\left(\tilde{\Xi}^{r}(\nu)\right)(x), \omega\right\rangle .
$$

As $\Theta^{r}$ is expressed in (7.6), we can also express $\Phi_{L}$ by

$$
\Phi_{L}(\nu)(x)=\nu \square x=(\iota \otimes \nu) V(x \otimes 1) V^{*}
$$

for $\nu \in L U C(\mathbb{G})^{*}$ and $x \in \mathcal{B}\left(L_{2}(\mathbb{G})\right)$. Therefore, the commutative diagram below is clearly dual to the diagram (7.1):



Proposition 7.2. Let $\mathbb{G}$ be a locally compact quantum group. then the following are equivalent:

(1) $\tilde{\iota}^{r}: U C Q^{r}\left(L_{1}(\mathbb{G})\right) \rightarrow L U C(\mathbb{G})$ is a bounded linear isomorphism;

(2) $\Xi^{r}: \operatorname{LUC}(\mathbb{G})^{*} \rightarrow U C Q^{r}\left(L_{1}(\mathbb{G})\right)^{*}$ is surjective;

(3) $\Phi_{L}: L U C(\mathbb{G})^{*} \rightarrow \mathcal{C B}_{\mathcal{T}\left(L_{2}(\mathbb{G})\right)}\left(\mathcal{B}\left(L_{2}(\mathbb{G})\right)\right)$ is bounded from below.

Proof. Recall that the range of $\Xi^{r}$ is weak*-dense in $U C Q^{r}\left(L_{1}(\mathbb{G})\right)^{*}$, and $U C Q^{r}\left(L_{1}(\mathbb{G})\right) \subseteq L U C(\mathbb{G})$ is norm dense. Thus we have $(1) \Leftrightarrow(2)$.

Note that $\Phi_{L}=\Theta^{r} \circ \Xi^{r}$, and $\Theta^{r}: U C Q^{r}\left(L_{1}(\mathbb{G})\right)^{*} \rightarrow \mathcal{C} \mathcal{B}_{\mathcal{T}\left(L_{2}(\mathbb{G})\right)}\left(\mathcal{B}\left(L_{2}(\mathbb{G})\right)\right)$ is completely isometric. Hence, we also have $(2) \Leftrightarrow(3)$.

Theorem 7.3. Let $\mathbb{G}$ be a co-amenable locally compact quantum group. Then the maps

$$
\begin{gathered}
\tilde{\iota}^{r}: U C Q^{r}\left(L_{1}(\mathbb{G})\right) \rightarrow L U C(\mathbb{G}), \quad \tilde{\Xi}^{r}: L U C(\mathbb{G})^{*} \rightarrow U C Q^{r}\left(L_{1}(\mathbb{G})\right)^{*}, \\
\text { and } \tilde{\Theta}^{r}: U C Q^{r}\left(L_{1}(\mathbb{G})\right)^{*} \rightarrow \mathcal{C} \mathcal{B}_{\mathcal{T}\left(L_{2}(\mathbb{G})\right)}\left(\mathcal{B}\left(L_{2}(\mathbb{G})\right)\right)
\end{gathered}
$$

are all completely isometric surjections. In this case, we also have

$$
U C Q^{r}\left(L_{1}(\mathbb{G})\right)=U C Q^{r}\left(L_{1}(\mathbb{G})\right) \triangleright \mathcal{T}\left(L_{2}(\mathbb{G})\right) .
$$

Proof. According to Proposition 6.5, the map $\Phi_{L}: L U C(\mathbb{G})^{*} \rightarrow \mathcal{C B}_{\mathcal{T}\left(L_{2}(\mathbb{G})\right)}\left(\mathcal{B}\left(L_{2}(\mathbb{G})\right)\right)$ is surjective and completely isometric. Since $\Phi_{L}=\Theta^{r} \circ \Xi^{r}$ and $\Theta^{r}$ is completely isometric, the map $\tilde{\Theta}^{r}$ must be onto $\mathcal{C B}_{\mathcal{T}\left(L_{2}(\mathbb{G})\right)}\left(\mathcal{B}\left(L_{2}(\mathbb{G})\right)\right)$, and the map $\tilde{\Xi}^{r}$ must be completely isometrically onto $U C Q^{r}\left(L_{1}(\mathbb{G})\right)^{*}$. In this situation, $\tilde{\iota}^{r}$ is also completely isometric and onto $L U C(\mathbb{G})$, and we can conclude from Corollary 5.5 that

$$
U C Q^{r}\left(L_{1}(\mathbb{G})\right)=L U C(\mathbb{G})=L U C(\mathbb{G}) \triangleright \mathcal{T}\left(L_{2}(\mathbb{G})\right)=U C Q^{r}\left(L_{1}(\mathbb{G})\right) \triangleright \mathcal{T}\left(L_{2}(\mathbb{G})\right)
$$


Remark 7.4. (i) Let $\mathbb{G}$ be a co-commutative locally compact quantum group. By [33, Theorem 4.1], we see that each of (1) - (3) in Proposition 7.2 is equivalent to $\mathbb{G}$ being co-amenable. The equivalence between (3) and the co-amenability of $\mathbb{G}$ can also be obtained by the fact that a locally compact group $G$ is amenable if and only if $\|\cdot\|_{A(G)}$ and $\|\cdot\|_{M_{c b} A(G)}$ are equivalent on $A(G)$ (cf. Losert [28] and Ruan [36]). It is still open whether this holds for general locally compact quantum groups (see also Remark 4.6(i)).

(ii) It is unknown, even for the commutative discrete quantum group $\mathbb{G}=L_{\infty}(\mathbb{Z})$, whether $\tilde{\Theta}^{r}$ or $\Phi_{L}$ is onto $\mathcal{C B}_{L_{\infty}(\hat{\mathbb{G}})}^{L_{\infty}(\mathbb{B})}\left(\mathcal{B}\left(L_{2}(\mathbb{G})\right)\right)$.

(iii) It is interesting to compare the diagrams (4.3) and (7.1). It is not clear whether there exists a complete contraction $\pi_{\rho}: Q^{r}\left(L_{1}(\mathbb{G})\right) \rightarrow U C Q^{r}\left(L_{1}(\mathbb{G})\right)$ such that $\tilde{\iota}^{r} \circ \pi_{\rho}(\omega)=\tilde{\rho}_{*}(\omega)\left(\omega \in Q^{r}\left(L_{1}(\mathbb{G})\right)\right)$, though, by Theorem 4.4 and Theorem 7.3, this is the case when $\mathbb{G}$ is co-amenable.

Recall that $\mathbb{G}$ is co-amenable if and only if $\operatorname{LUC}(\mathbb{G})^{*}$ is unital (cf. [14, Theorem 15]). Therefore, by Proposition 6.5, we have

$$
\begin{aligned}
\mathbb{G} \text { is co-amenable } & \Longleftrightarrow \Phi_{L} \text { is onto } \mathcal{C B}_{\mathcal{T}\left(L_{2}(\mathbb{G})\right)}\left(\mathcal{B}\left(L_{2}(\mathbb{G})\right)\right) \\
& \Longleftrightarrow \Theta^{r}\left(M_{c b}^{r}\left(L_{1}(\mathbb{G})\right)\right) \subseteq \Phi_{L}\left(L U C(\mathbb{G})^{*}\right) \\
& \Longleftrightarrow L U C(\mathbb{G})^{*} \text { is unital. }
\end{aligned}
$$

However, when we replace in the above $\Phi_{L}$ by $\tilde{\Theta}^{r}$, and $L U C(\mathbb{G})^{*}$ by $U C Q^{r}\left(L_{1}(\mathbb{G})\right)^{*}$, we are only able to obtain the three implications " $\Rightarrow$ ". We have one more implication as shown below.

Proposition 7.5. Let $\mathbb{G}$ be a locally compact quantum group. If $U C Q^{r}\left(L_{1}(\mathbb{G})\right)^{*}$ is unital, then $L_{1}(\mathbb{G})$ has a right approximate identity.

Proof. Let us identify $U C Q^{r}\left(L_{1}(\mathbb{G})\right)^{*}$ with its canonical image in $\mathcal{C B}_{\mathcal{T}\left(L_{2}(\mathbb{G})\right)}\left(\mathcal{B}\left(L_{2}(\mathbb{G})\right)\right)$ via $\tilde{\Theta}^{r}$. Suppose that $1 \in U C Q^{r}\left(L_{1}(\mathbb{G})\right)^{*}$. Since $L_{1}(\mathbb{G})$ is weak*-dense in $L U C(\mathbb{G})^{*}$ and $\Phi_{L}\left(L U C(\mathbb{G})^{*}\right)$ is weak*-dense in $U C Q^{r}\left(L_{1}(\mathbb{G})\right)^{*}$, there exists a net $\left\{f_{\alpha}\right\}$ in $L_{1}(\mathbb{G})$ such that $\Phi_{L}\left(f_{\alpha}\right) \rightarrow 1$ in the weak* topology on $U C Q^{r}\left(L_{1}(\mathbb{G})\right)^{*}$. In particular, for all $x \in L_{\infty}(\mathbb{G})$ and $f \in L_{1}(\mathbb{G})$, we have

$$
\left\langle x, f \star f_{\alpha}\right\rangle=\left\langle x \star f, f_{\alpha}\right\rangle=\left\langle\Phi_{L}\left(f_{\alpha}\right), x \star f\right\rangle \rightarrow\langle 1, x \star f\rangle=\langle x, f\rangle .
$$

Therefore, $\left\{f_{\alpha}\right\}$ is a weak right approximate identity of $L_{1}(\mathbb{G})$. By a standard convexity argument, we can obtain a right approximate identity of $L_{1}(\mathbb{G})$

Turning to the left-hand side theory, we can analogously define the complete contraction

$$
\tilde{\mathcal{S}}^{l}: \omega \otimes x \in \mathcal{T}\left(L_{2}(\mathbb{G})\right) \hat{\otimes} \mathcal{B}\left(L_{2}(\mathbb{G})\right) \rightarrow \omega \triangleleft x=\left\langle\iota \otimes \omega, W^{*}(1 \otimes x) W\right\rangle \in R U C(\mathbb{G}) .
$$

We let $U C Q^{l}\left(L_{1}(\mathbb{G})\right)$ denote the range space of $\tilde{\mathcal{S}}^{l}$ endowed with the operator space matrix norm from the quotient space $\mathcal{T}\left(L_{2}(\mathbb{G})\right) \hat{\otimes} \mathcal{B}\left(L_{2}(\mathbb{G})\right) / \operatorname{ker} \tilde{\mathcal{S}}^{l}$, and let $\tilde{\pi}^{l}: \mathcal{T}\left(L_{2}(\mathbb{G})\right) \hat{\otimes} \mathcal{B}\left(L_{2}(\mathbb{G})\right) \rightarrow U C Q^{l}\left(L_{1}(\mathbb{G})\right)$ be 
the complete quotient map. We can consider the left $\left(\mathcal{T}\left(L_{2}(\mathbb{G})\right), \triangleright\right)$-module structure on $U C Q^{l}\left(L_{1}(\mathbb{G})\right)$, and then obtain analogously a completely contractive Banach algebra structure on $U C Q^{l}\left(L_{1}(\mathbb{G})\right)^{*}$, which enjoys properties perfectly analogous to $U C Q^{r}\left(L_{1}(\mathbb{G})\right)^{*}$. The only difference is that the corresponding map $\tilde{\Theta}^{l}=\left(\tilde{\pi}^{l}\right)^{*}$ is now an anti-homomorphism, instead of a homomorphism.

Theorem 7.6. Let $\mathbb{G}$ be a locally compact quantum group. Then $U C Q^{l}\left(L_{1}(\mathbb{G})\right)^{*}$ is a completely contractive Banach algebra, and

$$
\tilde{\Theta}^{l}=\left(\tilde{\pi}^{l}\right)^{*}: U C Q^{l}\left(L_{1}(\mathbb{G})\right)^{*} \rightarrow \mathcal{C B}\left(\mathcal{B}\left(L_{2}(\mathbb{G})\right)\right)
$$

is a weak ${ }^{*}$-weak ${ }^{*}$ continuous completely isometric algebra anti-homomorphism satisfying

$$
\tilde{\Theta}^{l}\left(U C Q^{l}\left(L_{1}(\mathbb{G})\right)^{*}\right) \subseteq\left(\mathcal{T}\left(L_{2}(\mathbb{G})\right), \triangleright\right) \mathcal{C B}\left(\mathcal{B}\left(L_{2}(\mathbb{G})\right)\right) \cap \mathcal{C B}_{L_{\infty}\left(\mathbb{G}^{\prime}\right)}^{L_{\infty}(\mathbb{B})}\left(\mathcal{B}\left(L_{2}(\mathbb{G})\right)\right) .
$$

When $\mathbb{G}$ is co-amenable, we have

$$
U C Q^{l}\left(L_{1}(\mathbb{G})\right)=R U C(\mathbb{G}) \text { completely isometrically, }
$$

and

$U C Q^{l}\left(L_{1}(\mathbb{G})\right) \cong_{\left(\mathcal{T}\left(L_{2}(\mathbb{G})\right), \triangleright\right)} \mathcal{C B}\left(\mathcal{B}\left(L_{2}(\mathbb{G})\right)\right)$ completely isometrically and anti-isomorphically via $\tilde{\Theta}^{l}$.

\section{REFERENCES}

[1] S. Baaj and G. Skandalis, Unitaires multiplicatifs et dualité pour les produits croisés de $C^{*}$-algèbres, Ann. Sci. École Norm. Sup. 26 (1993), 425-488.

[2] E. Bédos, G. Murphy, and L. Tuset, Amenability and co-amenability of algebraic quantum groups, Int. J. Math. Math. Sci. 31 (2002), 577-601.

[3] E. Bédos and L. Tuset, Amenability and co-amenability for locally compact quantum groups, Internat. J. Math. 14 (2003), 865-884.

[4] D. Blecher and R. R. Smith, The dual of the Haagerup tensor product, J. London Math. Soc. 45 (1992), 126-144.

[5] M. Bożejko, Remark on Herz-Schur multipliers on free groups, Math. Ann. 258 (1981/82), 11-15.

[6] E. G. Effros and A. Kishimoto, Module maps and Hochschild-Johnson cohomology, Indiana Univ. Math. J. 36 (1987), $257-276$.

[7] E. G. Effros, J. Kraus, and Z.-J. Ruan, ...,

[8] M. Enock and J.-M. Schwartz, Kac algebras and duality of locally compact groups, Springer-Verlag, Berlin-Heidelberg, 1992.

[9] F. Ghahramani, A. T.-M. Lau, and V. Losert, Isometric isomorphisms between Banach algebras related to locally compact groups, Trans. Amer. Math. Soc. 321 (1990), 273-283.

[10] E. E. Granirer, Weakly almost periodic and uniformly continuous functionals on the Fourier algebra of any locally compact group, Trans. Amer. Math. Soc. 189 (1974), 371-382.

[11] U. Haagerup, Decomposition of completely bounded maps on operator algebras, manuscript, 1980.

[12] U. Haagerup and J. Kraus,Approximation properties for group $C^{*}$-algebras and group von Neumann algebras, Trans. Amer. Math. Soc. 344 (1994), 667-699.

[13] Z. Hu, M. Neufang, and Z.-J. Ruan, Multipliers on a new class of Banach algebras, locally compact quantum groups, and topological centres, Proc. London Math. Soc., to appear.

[14] Z. Hu, M. Neufang, and Z.-J. Ruan, On topological centre problems and SIN quantum groups, J. Funct. Anal. 257 (2009), 610-640. 
[15] Z. Hu, M. Neufang, and Z.-J. Ruan, On module homomorphisms and Arens irregularity properties, preprint.

[16] B. E. Johnson An introduction to double centralizers, Proc. London Math. Soc. 14 (1964), $299-320$.

[17] M. Junge, M. Neufang, and Z.-J. Ruan, A representation theorem for locally compact quantum groups, Internat. J. Math. 20 (2009), 377-400.

[18] M. Kalantar and M. Neufang, Convolution algebras over locally compact quantum groups, manuscript in preparation.

[19] J. Kraus and Z.-J. Ruan, Multipliers of Kac algebras, Internat. J. Math. 8 (1997), 213-248.

[20] J. Kustermans and S. Vaes, Locally compact quantum groups, Ann. Sci. Ecole Norm. Sup. 33 (2000), 837-934.

[21] J. Kustermans and S. Vaes, Locally compact quantum groups in the von Neumann algebraic setting, Math. Scand. 92 (2003), 68-92.

[22] A. T.-M. Lau, Continuity of Arens multiplication on the dual space of bounded uniformly continuous functions on locally compact groups and topological semigroups, Math. Proc. Cambridge Philos. Soc. 99 (1986), $273-283$.

[23] A. T.-M. Lau, Uniformly continuous functionals on Banach algebras, Colloq. Math. 51 (1987), $195-205$.

[24] A. T.-M. Lau and V. Losert, The $C^{*}$-algebra generated by operators with compact support on a locally compact group, J. Funct. Anal. 112 (1993), 1-30.

[25] A. T.-M. Lau and V. Losert, The centre of the second conjugate algebra of the Fourier algebra for infinite products of groups, Math. Proc. Cambridge Philos. Soc. 138 (2005), 27-39.

[26] A. T.-M. Lau and K. McKennon, Isomorphisms of locally compact groups and Banach algebras, Proc. Amer. Math. Soc. 79 (1980), 55-58.

[27] V. Losert, Properties of the Fourier algebra that are equivalent to amenability, Proc. Amer. Math. Soc. 92 (1984), $549-554$.

[28] V. Losert, Fourier algebra unt mittelbare gruppen, Lectures at Heidelberg University, 1985.

[29] M. Neufang, Abstrakte harmonische Analyse und Modulhomomorphismen über von Neumann-Algebren, Ph.D. thesis, Universität des Saarlandes, 2000.

[30] M. Neufang, Z.-J. Ruan, and N. Spronk, Completely isometric representations of $M_{c b} A(G)$ and $U C B(\hat{G})^{*}$, Trans. Amer. Math. Soc. 360 (2008), 1133-1161.

[31] M. Neufang and V. Runde, Harmonic operators: the dual perspective, Math. Z. 255 (2007), 669-690.

[32] A. Yu. Pirkovskii, Biprojectivity and biflatness for convolution algebras of nuclear operators, Canad. Math. Bull. 47 (2004), 445-455.

[33] A.-M. Popa and Z.-J. Ruan, Uniformly complete quotient space UCQ $(G)$ and completely isometric representations of $U C Q(G)^{*}$ on $B\left(L_{2}(G)\right)$, Proc. Amer. Math. Soc. 134 (2005), 1223-1235.

[34] M. Ramezanpour and H. R. E. Vishki, Module homomorphisms and multipliers on locally compact quantum groups, preprint, 2009.

[35] Z.-J. Ruan, Amenability of Hopf von Neumann algebras and Kac algebras, J. Funct. Anal. 139 (1996), $466-499$.

[36] Z.-J. Ruan, A characterization of amenable groups, manuscript, 1996.

[37] V. Runde, Uniform continuity over locally compact quantum groups, J. London Math. Soc., to appear.

[38] P. Salmi, Quantum semigroup compactifications and left uniformly continuous functionals, preprint, 2008.

[39] S. Vaes, Locally compact quantum groups, Ph.D. thesis, Katholieke Universitiet Leuven, Leuven, 2001.

[40] J. G. Wendel, Left centralizers and isomorphisms of group algebras, Pacific Math. J. 2 (1952), $251-261$.

Department of Mathematics and Statistics, University of Windsor, Windsor, Ontario, Canada N9B 3P4

E-mail address: zhiguohu@uwindsor.ca

School of Mathematics and Statistics, Carleton University, Ottawa, Ontario, Canada K1S 5B6

E-mail address: mneufang@math.carleton.ca

Department of Mathematics, University of Illinois, Urbana, IL 61801, USA

E-mail address: ruan@math.uiuc.edu 